\title{
Stability in a Simple Food Chain System with Michaelis-Menten Functional Response and Nonlocal Delays
}

\author{
Wenzhen Gan, ${ }^{1}$ Canrong Tian, ${ }^{2}$ Qunying Zhang, ${ }^{3}$ and Zhigui Lin ${ }^{3}$ \\ ${ }^{1}$ School of Mathematics and Physics, Jiangsu Teachers University of Technology, Changzhou 213001, China \\ ${ }^{2}$ Basic Department, Yancheng Institute of Technology, Yangcheng 224003, China \\ ${ }^{3}$ School of Mathematical Science, Yangzhou University, Yangzhou 225002, China \\ Correspondence should be addressed to Zhigui Lin; zglin68@hotmail.com
}

Received 26 April 2013; Accepted 8 July 2013

Academic Editor: Rodrigo Lopez Pouso

Copyright (C) 2013 Wenzhen Gan et al. This is an open access article distributed under the Creative Commons Attribution License, which permits unrestricted use, distribution, and reproduction in any medium, provided the original work is properly cited.

\begin{abstract}
This paper is concerned with the asymptotical behavior of solutions to the reaction-diffusion system under homogeneous Neumann boundary condition. By taking food ingestion and species' moving into account, the model is further coupled with MichaelisMenten type functional response and nonlocal delay. Sufficient conditions are derived for the global stability of the positive steady state and the semitrivial steady state of the proposed problem by using the Lyapunov functional. Our results show that intraspecific competition benefits the coexistence of prey and predator. Furthermore, the introduction of Michaelis-Menten type functional response positively affects the coexistence of prey and predator, and the nonlocal delay is harmless for stabilities of all nonnegative steady states of the system. Numerical simulations are carried out to illustrate the main results.
\end{abstract}

\section{Introduction}

The overall behavior of ecological systems continues to be of great interest to both applied mathematicians and ecologists. Two species predator-prey models have been extensively investigated in the literature. But recently more and more attention has been focused on systems with three or more trophic levels. For example, the predator-prey system for three species with Michaelis-Menten type functional response was studied by many authors [1-4]. However, the systems in [1-4] are either with discrete delay or without delay or without diffusion. In view of individuals taking time to move, spatial dispersal was dealt with by introducing diffusion term to corresponding delayed ODE model in previous literatures, namely, adding a Laplacian term to the ODE model. In recent years, it has been recognized that there are modelling difficulties with this approach. The difficulty is that diffusion and time delay are independent of each other, since individuals have not been at the same point at previous times. Britton [5] made a first comprehensive attempt to address this difficulty by introducing a nonlocal delay; that is, the delay term involves a weighted-temporal average over the whole of the infinite domain and the whole of the previous times.

There are many results for reaction-diffusion equations with nonlocal delays [5-18]. The existence and stability of traveling wave fronts were studied in reaction-diffusion equations with nonlocal delay [5-9]. The stability of impulsive cellular neural networks with time varying was discussed in [10] by means of new Poincare integral inequality. The asymptotic behavior of solutions of the reaction-diffusion equations with nonlocal delay was investigated in $[11,12]$ by using an iterative technique and in [13-15] by the Lyapunov functional. The stability and Hopf bifurcation were discussed in [16] for a diffusive logistic population model with nonlocal delay effect.

Motivated by the work above, we are concerned with the following food chain model with Michaelis-Menten type functional response:

$$
\frac{\partial u_{1}}{\partial t}-d_{1} \Delta u_{1}=u_{1}\left(a_{1}-a_{11} u_{1}-\frac{a_{12} u_{2}}{m_{1}+u_{1}}\right)
$$




$$
\begin{gathered}
\frac{\partial u_{2}}{\partial t}-d_{2} \Delta u_{2} \\
=u_{2}\left(-a_{2}+a_{21} \int_{\Omega} \int_{-\infty}^{t} \frac{u_{1}(s, y)}{m_{1}+u_{1}(s, y)}\right. \\
\times K_{1}(x, y, t-s) d s d y \\
\left.-a_{22} u_{2}-\frac{a_{23} u_{3}}{m_{2}+u_{2}}\right), \\
\frac{\partial u_{3}}{\partial t}-d_{3} \Delta u_{3} \\
=u_{3}\left(-a_{3}+a_{32} \int_{\Omega}^{t} \frac{u_{2}(s, y)}{\int_{-\infty}+u_{2}(s, y)}\right. \\
\times K_{2}(x, y, t-s) d s d y \\
\left.-a_{33} u_{3}\right),
\end{gathered}
$$

for $t>0, x \in \Omega$ with homogeneous Neumann boundary conditions

$$
\frac{\partial u_{1}}{\partial v}=\frac{\partial u_{2}}{\partial v}=\frac{\partial u_{3}}{\partial v}=0, \quad t>0, x \in \partial \Omega,
$$

and initial conditions

$$
\begin{array}{ll}
u_{i}(t, x)=\phi_{i}(t, x) \geq 0 & (i=1,2),(t, x) \in(-\infty, 0] \times \Omega, \\
u_{3}(0, x)=\phi_{3}(x) \geq 0, \quad x \in \Omega,
\end{array}
$$

where $\phi_{i}$ is bounded, Hölder continuous function and satisfies $\partial \phi_{i} / \partial v=0(i=1,2,3)$ on $(-\infty, 0] \times \partial \Omega$. Here, $\Omega$ is a bounded domain in $\mathbb{R}^{n}$ with smooth boundary $\partial \Omega$ and $\partial / \partial \nu$ is the outward normal derivative on $\partial \Omega . u_{i}(t, x)$ represents the density of the $i$ th species (prey, predator, and top predator resp.) at time $t$ and location $x$ and thus only nonnegative $u_{i}(t, x)$ is of interest. The parameter $a_{1}$ is the intrinsic growth rate of the prey, and $a_{2}$ and $a_{3}$ are the death rates of the predator and top-predator. $a_{i i}$ is the intraspecific competitive rate of the $i$ th species. $a_{12}$ is the maximum predation rate. $a_{23}$ and $a_{32}$ are the efficiencies of food utilization of the predator and top predator, respectively. We assume the predator and top predator show the Michaelis-Menten (or Holling type II) functional response with $u_{1} /\left(m_{1}+u_{1}\right)$ and $u_{2} /\left(m_{2}+u_{2}\right)$, respectively, where $m_{1}$ and $m_{2}$ are half-saturation constants. For a through biological background of similar models, see $[18,19]$. As our most knowledge, the tritrophic food chain model has been found to have many interesting biological properties, such as the coexistence and the Hopf bifurcation. However, the effect of nonlocal time delays on the coexistence has not been reported. Our paper mainly concerns this perspective.

Additionally, $\int_{\Omega} \int_{-\infty}^{t}\left(u_{i}(s, y) /\left(m_{i}+u_{i}(s, y)\right)\right) K_{i}(x, y, t-$ $s) d s d y(i=1,2)$ represents the nonlocal delay due to the ingestion of predator; that is, mature adult predator can only contribute to the production of predator biomass. The boundary condition in (2) implies that there is no migration across the boundary of $\Omega$.

The main purpose of this paper is to study the global asymptotic behavior of the solution of system (1)-(3). The preliminary results are presented in Section 2. Section 3 contains sufficient conditions for the global asymptotic behaviors of the equilibria of system (1)-(3) by means of the Lyapunov functional. Numerical simulations are carried out to show the feasibility of the conditions in Theorems 8-10 in Section 4. Finally, a brief discussion is given to conclude this work.

\section{Preliminary Results}

In this section, we present several preliminary results that will be employed in the sequel.

Lemma 1 (see [3]). Let $a$ and $b$ be positive constants. Assume that $\phi, \varphi \in C^{1}(a,+\infty), \varphi(t) \geq 0$, and $\phi$ is bounded from below. If $\phi^{\prime}(t) \leq-b \varphi(t)$ and $\varphi^{\prime}(t) \leq K$ in $[a,+\infty)$ for some positive constant $K$, then $\lim _{t \rightarrow+\infty} \varphi(t)=0$.

The following lemma is the Positivity Lemma in [20].

Lemma 2. Let $u_{i} \in C([0, T] \times \bar{\Omega}) \bigcap C^{1,2}((0, T] \times \Omega)(i=$ $1,2,3)$ and satisfy

$$
\begin{aligned}
u_{i t}-d_{i} \Delta u_{i} \geq & \sum_{j=1}^{3} b_{i j}(t, x) u_{j}(t, x) \\
& +\sum_{j=1}^{3} c_{i j} u_{j}\left(t-\tau_{j}, x\right) \quad \text { in }(0, T] \times \Omega, \\
\frac{\partial u_{i}(t, x)}{\partial \nu} \geq 0 \quad \text { on }(0, T] \times \partial \Omega, & \text { in }\left[-\tau_{i}, 0\right] \times \Omega,
\end{aligned}
$$

where $b_{i j}, c_{i j} \in C([0, T] \times \bar{\Omega}), u_{i t}=\left(u_{i}\right)_{t}$. If $b_{i j} \geq 0$ for $j \neq i$ and $c_{i j} \geq 0$ for all $i, j=1,2,3$. Then $u_{i}(t, x) \geq 0$ on $[0, T] \times \bar{\Omega}$. Moreover, if the initial function is nontrivial, then $u_{i}>0$ in $(0, T] \times \bar{\Omega}$.

Lemma 3 (see [20]). Let $\widehat{\mathbf{c}}$ and $\widetilde{\mathbf{c}}$ be a pair of constant vector satisfying $\widetilde{\mathbf{c}} \geq \widehat{\mathbf{c}}$ and let the reaction functions satisfy local Lipschitz condition with $\Lambda=\langle\widehat{\mathbf{c}}, \widetilde{\mathbf{c}}\rangle$. Then system (1)-(3) admits a unique global solution $\mathbf{u}(t, x)$ such that

$$
\widehat{\mathbf{c}} \leq \mathbf{u}(t, x) \leq \widetilde{\mathbf{c}}, \quad \forall t>0, x \in \bar{\Omega},
$$

whenever $\widehat{\mathbf{c}} \leq \phi(t, x) \leq \widetilde{\mathbf{c}},(t, x) \in(-\infty, 0] \times \Omega$.

The following result was obtained by the method of upper and lower solutions and the associated iterations in [21]. 
Lemma 4 (see [21]). Let $u(t, x) \in C([0, \infty) \times \bar{\Omega}) \cap$ $C^{2,1}((0, \infty) \times \Omega)$ be the nontrivial positive solution of the system

$$
\begin{aligned}
& u_{t}-d \Delta u=B u(t-\tau, x) \pm A_{1} u(t, x) \\
& \quad-A_{2} u^{2}(t, x), \quad(t, x) \in(0, \infty) \times \Omega, \\
& \begin{array}{l}
\frac{\partial u}{\partial v}=0, \quad(t, x) \in(0, \infty) \times \partial \Omega, \\
u(t, x)=\phi(t, x) \geq 0, \quad(t, x) \in(-\tau, 0) \times \Omega,
\end{array}
\end{aligned}
$$

where $A_{1} \geq 0, B, A_{2}, \tau>0$. The following results hold:

(1) if $B \pm A_{1}>0$, then $u \rightarrow\left(B \pm A_{1}\right) / A_{2}$ uniformly on $\bar{\Omega}$ as $t \rightarrow \infty$;

(2) if $B \pm A_{1} \leq 0$, then $u \rightarrow 0$ uniformly on $\bar{\Omega}$ as $t \rightarrow \infty$.

Throughout this paper, we assume that

$$
\begin{gathered}
K_{i}(x, y, t)=G_{i}(x, y, t) k_{i}(t), \quad x, y \in \Omega, k_{i}(t) \geq 0 ; \\
\int_{\Omega} G_{i}(x, y, t) d x=\int_{\Omega} G_{i}(x, y, t) d y=1, \quad t \geq 0 ; \\
\int_{0}^{+\infty} k_{i}(t) d t=1, \quad t k_{i}(t) \in L^{1}((0,+\infty) ; R),
\end{gathered}
$$

where $G_{i}(x, y, t)$ is a nonnegative function, which is continuous in $(x, y) \in \bar{\Omega} \times \bar{\Omega}$ for each $t \in[0,+\infty)$ and measurable in $t \in[0,+\infty)$ for each pair $(x, y) \in \bar{\Omega} \times \bar{\Omega}$.

Now we prove the following propositions which will be used in the sequel.

Proposition 5. For any nonnegative initial function, the corresponding solution of system (1)-(3) is nonnegative.

Proof. Suppose that $\left(u_{1}, u_{2}, u_{3}\right)$ is a solution and satisfies $u_{i} \in$ $C([0, T) \times \bar{\Omega}) \cap C^{1,2}((0, T) \times \Omega)$ with $T \leq+\infty$. Choose $0<$ $\tau<T$. Then

$$
\begin{aligned}
& u_{1 t}-d_{1} \Delta u_{1}=A_{1} u_{1}, \quad 0<t<\tau, \quad x \in \Omega, \\
& u_{2 t}-d_{2} \Delta u_{2}=A_{2} u_{2}, \quad 0<t<\tau, \quad x \in \Omega, \\
& u_{3 t}-d_{3} \Delta u_{3}=A_{3} u_{3}, \quad 0<t<\tau, \quad x \in \Omega, \\
& \frac{\partial u_{i}}{\partial \nu}=0, \quad 0<t<\tau, \quad x \in \partial \Omega, \\
& u_{i}(t, x) \geq 0 \quad(i=1,2,3), \quad t \leq 0, \quad x \in \bar{\Omega},
\end{aligned}
$$

where

$$
\begin{aligned}
& A_{1}=a_{1}-a_{11} u_{1}-\frac{a_{12} u_{2}}{m_{1}+u_{1}}, \\
& A_{2}=-a_{2}+a_{21} \int_{\Omega} \int_{-\infty}^{t} \frac{u_{1}(s, y)}{m_{1}+u_{1}(s, y)} \\
& \times K_{1}(x, y, t-s) d s d y \\
& -a_{22} u_{2}-\frac{a_{23} u_{3}}{m_{2}+u_{3}}, \\
& A_{3}=-a_{3}+a_{32} \int_{\Omega} \int_{-\infty}^{t} \frac{u_{2}(s, y)}{m_{2}+u_{2}(s, y)} \\
& \times K_{2}(x, y, t-s) d s d y-a_{33} u_{3} .
\end{aligned}
$$

It follows from Lemma 2 that $u_{i} \geq 0,(t, x) \in[0, \tau] \times \bar{\Omega}$. Due to the arbitrariness of $\tau$, we have $u_{i} \geq 0$ for $(t, x) \in[0, T) \times$ $\bar{\Omega}$.

Proposition 6. System (1)-(3) with initial functions $\phi_{i}$ admits a unique global solution $\left(u_{1}, u_{2}, u_{3}\right)$ satisfying $0 \leq u_{i}(t, x) \leq$ $M_{i}$ for $i=1,2,3$, where $M_{i}$ is defined as

$$
\begin{aligned}
& M_{1}=\max \left\{\frac{a_{1}}{a_{11}},\left\|\phi_{1}(t, x)\right\|_{L^{\infty}((-\infty, 0] \times \bar{\Omega})}\right\}, \\
& M_{2}=\max \left\{\frac{\left(a_{21}-a_{2}\right) M_{1}-m_{1} a_{2}}{a_{22}\left(m_{1}+M_{1}\right)},\left\|\phi_{2}(t, x)\right\|_{L^{\infty}((-\infty, 0] \times \bar{\Omega})}\right\}, \\
& M_{3}=\max \left\{\frac{\left(a_{32}-a_{3}\right) M_{2}-m_{2} a_{3}}{a_{22}\left(m_{2}+M_{2}\right)},\left\|\phi_{3}(x)\right\|_{L^{\infty}(\bar{\Omega})}\right\} .
\end{aligned}
$$

Proof. It follows from standard PDE theory that there exists a $T>0$ such that problem (1)-(3) admits a unique solution in $[0, T) \times \bar{\Omega}$. From Lemma 2 we know that $u_{i}(t, x) \geq 0$ for $(t, x) \in[0, T) \times \bar{\Omega}$. Considering the first equation in (1), we have

$$
\frac{\partial u_{1}}{\partial t}-d_{1} \Delta u_{1} \leq u_{1}\left(a_{1}-a_{11} u_{1}\right)
$$

Using the maximum principle gives that $u_{1} \leq M_{1}$. In a similar way, we have $u_{i} \leq M_{i}$ for $i=2,3$. It is easy to see that $(0,0,0)$ and $\left(M_{1}, M_{2}, M_{3}\right)$ are a pair of coupled upper and lower solutions to system (1)-(3) from the direct computation. In virtue of Lemma 3, system (1)-(3) admits a unique global solution $\left(u_{1}, u_{2}, u_{3}\right)$ satisfying $0 \leq u_{i}(t, x) \leq M_{i}$ for $i=1,2,3$. In addition, if $\phi_{1}(t, x), \phi_{2}(t, x), \phi_{3}(x),(t, x) \in(-\infty, 0] \times \Omega$ is nontrivial, it follows from the strong maximum principle that $u_{i}(t, x)>0(i=1,2,3)$ for all $t>0, x \in \bar{\Omega}$.

\section{Global Stability}

In this section, we study the asymptotic behavior of the equilibrium of system (1)-(3). In the beginning, we show the existence and uniqueness of positive steady state. 


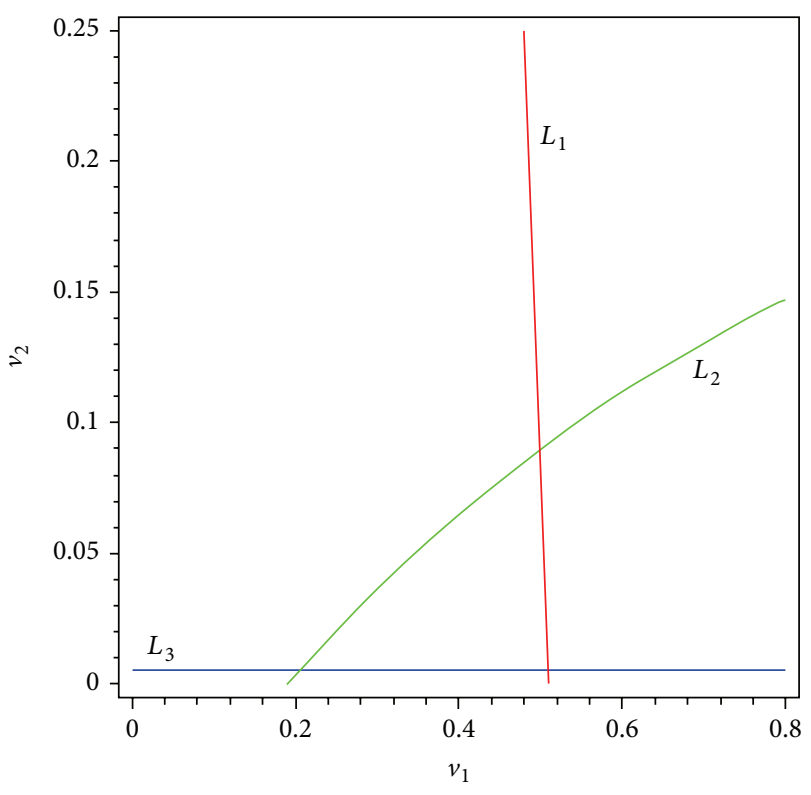

FIGURE 1: Graphs of the equations in (12). $L_{3}$ is the boundary condition $\left(a_{3}=a_{32} v_{2} /\left(m_{2}+v_{2}\right)\right)$ and $L_{2}$ corresponds to $-a_{2}+$ $a_{21}\left(v_{1} /\left(m_{1}+v_{1}\right)\right)-a_{22} v_{2}=0$, while $L_{1}$ corresponds to $a_{1}-a_{11} v_{1}-$ $a_{12} v_{2} /\left(m_{1}+v_{1}\right)=0$. Intersection point between $L_{1}$ and $L_{2}$ gives the positive solution $\left(v_{1}^{*}, v_{2}^{*}\right)$ to (12). Parameter values are listed in the example in Section 4.

Let us consider the following equations:

$$
\begin{aligned}
& a_{1}-a_{11} v_{1}-\frac{a_{12} v_{2}}{m_{1}+v_{1}}=0, \\
& -a_{2}+\frac{a_{21} v_{1}}{m_{1}+v_{1}}-a_{22} v_{2}=0 .
\end{aligned}
$$

A direct computation shows that the above equations have only one positive solution $\left(v_{1}^{*}, v_{2}^{*}\right)$ if and only if

$$
H_{1}: a_{1}\left(a_{21}-a_{2}\right)>m_{1} a_{2} a_{11}
$$

is satisfied. Taking $H_{1}$ into account, we consider the equation $a_{3}=a_{32} v_{2}^{*} /\left(m_{2}+v_{2}^{*}\right)$, which corresponds to $L_{3}$ in Figure 1 .

It is clear that if $a_{3}<a_{32} v_{2}^{*} /\left(m_{2}+v_{2}^{*}\right)$, the intersection point $\left(v_{1}^{*}, v_{2}^{*}\right)$ always lies in $L_{3}$. Let

$$
H_{2}: a_{3} m_{2}<\left(a_{32}-a_{3}\right) v_{2}^{*} \text {. }
$$

Suppose that $H_{1}$ and $H_{2}$ hold. We take $u_{3}$ as a parameter and consider the following system:

$$
\begin{aligned}
& a_{1}-a_{11} u_{1}-\frac{a_{12} u_{2}}{m_{1}+u_{1}}=0, \\
& -a_{2}+\frac{a_{21} u_{1}}{m_{1}+u_{1}}-a_{22} u_{2}-\frac{a_{23} u_{3}}{m_{2}+u_{2}}=0, \\
& -a_{3}+\frac{a_{32} u_{2}}{m_{2}+u_{2}}-a_{33} u_{3}=0 .
\end{aligned}
$$

When the parameter $u_{3}$ is sufficiently small, the first two equations in (15) can be approximated by (12). Moreover, by continuously increasing the value of $u_{3}, L_{3}$ goes up, and meanwhile the intersection point between $L_{1}$ and $L_{2}$ also goes up. However, $L_{3}$ goes up faster than $L_{2}$, while $L_{1}$ keeps still. In other words, there exists a critical value $u_{3}$ such that the intersection point lies in $L_{3}$, which implies that there is a unique positive solution $E^{*}\left(u_{1}^{*}, u_{2}^{*}, u_{3}^{*}\right)$ to (15), or equivalently (1)-(3).

Lemma 7. Assume that $\mathrm{H}_{2}$ and $\mathrm{G}_{2}$ hold, and then the positive steady state $E^{*}$ of system (1)-(3) is locally asymptotically stable.

Proof. We get the local stability of $E^{*}$ by performing a linearization and analyzing the corresponding characteristic equation. Similarly as in [22], let $0<\mu_{1}<\mu_{2}<\mu_{3}<$ $\ldots$ be the eigenvalues of $-\Delta$ on $\Omega$ with the homogeneous Neumann boundary condition. Let $E\left(\mu_{i}\right)$ be the eigenspace corresponding to $\mu_{i}$ in $C^{1}(\bar{\Omega})$, for $i=1,2,3, \ldots$ Let

$$
\mathbf{X}=\left\{\mathbf{u}=\left(u_{1}, u_{2}, u_{3}\right) \in\left[C^{1}(\bar{\Omega})\right]^{3} \partial_{\eta} \mathbf{u}=0, x \in \partial \Omega\right\},
$$

$\left\{\varphi_{i j}, j=1, \ldots, \operatorname{dim} E\left(\mu_{i}\right)\right\}$ be an orthonormal basis of $E\left(\mu_{i}\right)$, and $\mathbf{X}_{i j}=\left\{c \cdot \varphi_{i j} \mid c \in R^{3}\right\}$. Then

$$
\mathbf{X}_{i}=\bigoplus_{j=1}^{\operatorname{dim} E\left(\mu_{i}\right)} \mathbf{x}_{i j}, \quad \mathbf{X}=\bigoplus_{i=1}^{\infty} \mathbf{x}_{i}
$$

Let $D=\operatorname{diag}\left(D_{1}, D_{2}, D_{3}\right)$ and $\mathbf{v}=\left(v_{1}, v_{2}, v_{3}\right), v_{i}=$ $u_{i}-u_{i}^{*},(i=1,2,3)$. Then the linearization of $(1)$ is $\mathbf{v}_{t}=L \mathbf{v}=D \Delta \mathbf{v}+\mathbf{F}_{\mathbf{v}}\left(\mathbf{E}^{*}\right) \mathbf{v}, \mathbf{F}_{\mathbf{v}}\left(\mathbf{E}^{*}\right) \mathbf{v}=\left\{c_{i j}\right\}$, where $c_{i i}=b_{i i} v_{i}, i=1,2,3, c_{12}=b_{12} v_{2}, c_{23}=$ $b_{23} v_{3}, c_{21}=b_{21} \int_{\Omega} \int_{-\infty}^{t} v_{1}(s, y) K_{1}(x, y, t-s) d s d y$, and $c_{32}=$ $b_{32} \int_{\Omega} \int_{-\infty}^{t} v_{2}(s, y) K_{2}(x, y, t-s) d s d y$. The coefficients $b_{i j}$ are defined as follows:

$$
\begin{aligned}
& b_{11}=-a_{11} u_{1}^{*}+\frac{a_{12} u_{1}^{*} u_{2}^{*}}{\left(m_{1}+u_{1}^{*}\right)^{2}}, \\
& b_{12}=-\frac{a_{12} u_{1}^{*}}{m_{1}+u_{1}^{*}}, \quad b_{13}=0, \\
& b_{21}=\frac{a_{21} m_{1} u_{2}^{*}}{\left(m_{1}+u_{1}^{*}\right)^{2}}, \\
& b_{22}=-a_{22} u_{2}^{*}+\frac{a_{23} u_{2}^{*} u_{3}^{*}}{\left(m_{2}+u_{2}^{*}\right)^{2}}, \quad b_{23}=-\frac{a_{23} u_{2}^{*}}{m_{2}+u_{2}^{*}}, \\
& b_{31}=0, \quad b_{32}=\frac{a_{32} m_{2} u_{3}^{*}}{\left(m_{2}+u_{2}^{*}\right)^{2}}, \quad b_{33}=-a_{33} u_{3}^{*} .
\end{aligned}
$$

Since $\mathbf{X}_{i}$ is invariant under the operator $L$ for each $i \geq 1$, then the operator $L$ on $\mathbf{X}_{i}$ is $\mathbf{v}_{t}=L \mathbf{v}=D \mu_{i} \mathbf{v}+\mathbf{F}_{\mathbf{v}}\left(\mathbf{E}^{*}\right) \mathbf{v}$. Let $v_{i}=$ $c_{i} e^{\lambda t}(i=1,2,3)$ and we can get the characteristic equation 
$\varphi_{i}(\lambda)=\lambda^{3}+A_{i} \lambda^{2}+B_{i} \lambda+C_{i}=0$, where $A_{i}, B_{i}$, and $C_{i}$ are defined as follows:

$$
\begin{aligned}
A_{i}= & \left(d_{1}+d_{2}+d_{3}\right) \mu_{i}-b_{11}-b_{22}-b_{33}, \\
B_{i}= & \left(d_{1} d_{2}+d_{2} d_{3}+d_{3} d_{1}\right) \mu_{i}^{2} \\
+ & {\left[d_{3}\left(-b_{11}-b_{22}\right)+d_{1}\left(-b_{33}-b_{22}\right)\right.} \\
& \left.+d_{2}\left(-b_{11}-b_{33}\right)\right] \mu_{i} \\
+ & b_{11} b_{22}+b_{11} b_{33}+b_{22} b_{33} \\
- & b_{12} b_{21} \int_{0}^{\infty} k_{1}(s) e^{-\lambda s} d s-b_{23} b_{32} \int_{0}^{\infty} k_{2}(s) e^{-\lambda s} d s, \\
C_{i}= & d_{1} d_{2} d_{3} \mu_{i}^{3}+\left[-b_{33} d_{1} d_{2}-b_{11} d_{2} d_{3}-b_{22} d_{1} d_{3}\right] \mu_{i}^{2} \\
+ & {\left[b_{11} b_{22} d_{3}+b_{11} b_{33} d_{2}+b_{22} b_{33} d_{1}+b_{22} b_{33} d_{1}\right.} \\
& \quad-d_{3} b_{12} b_{21} \int_{0}^{\infty} k_{1}(s) e^{-\lambda s} d s \\
& \left.\left.\quad-b_{1} b_{23} b_{32} \int_{0}^{\infty} k_{2}(s) e^{-\lambda s} d s\right] \mu_{i} b_{32} \int_{0}^{\infty} k_{2}(s) e^{-\lambda s} d s\right] . \\
+ & -b_{11} b_{22} b_{33}+b_{12} b_{21} b_{33} \int_{0}^{\infty} k_{1}(s) e^{-\lambda s} d s
\end{aligned}
$$

It is easy to see that $b_{12}, b_{23}, b_{33}<0$ and $b_{21}, b_{32}>0$. It follows from assumption $G_{1}$ and $G_{2}$ that $a_{11}<0, a_{22}<0$. So $A_{i}>0, B_{i}>0, C_{i}>0$ and $A_{i} B_{i}-C_{i}>0$ for $i \geq 1$ from the direct calculation. According to the Routh-Hurwitz criterion, the three roots $\lambda_{i, 1}, \lambda_{i, 2}, \lambda_{i, 3}$ of $\varphi_{i}(\lambda)=0$ all have negative real parts.

By continuity of the roots with respect to $\mu_{i}$ and RouthHurwitz criterion, we can conclude that there exists a positive constant $\varepsilon$ such that

$$
\operatorname{Re}\left\{\lambda_{i, 1}\right\}, \operatorname{Re}\left\{\lambda_{i, 2}\right\}, \operatorname{Re}\left\{\lambda_{i, 3}\right\} \leq-\varepsilon, \quad i \geq 1 .
$$

Consequently, the spectrum of $L$, consisting only of eigenvalues, lies in $\{\operatorname{Re} \lambda \leq-\varepsilon\}$. It is easy to see that $\mathbf{E}^{*}$ is locally asymptotically stable and follows from Theorem 5.1.1 of [23].

Theorem 8. Assume that

$$
H_{3}: \frac{a_{1}\left(a_{21}-a_{2}\right)}{m_{1} a_{2}}>a_{11}>\frac{a_{12} u_{2}^{*}}{m_{1}^{2}},
$$

$\mathrm{H}_{2}$ and $\mathrm{G}_{2}$ hold, and the positive steady state $E^{*}$ of system (1)(3) with nontrivial initial function is globally asymptotically stable.
Proof. It is easy to see that the equations in (1) can be rewritten as

$$
\begin{aligned}
\frac{\partial u_{1}}{\partial t}-d_{1} \Delta u_{1}=u_{1}[ & -a_{11}\left(u_{1}-u_{1}^{*}\right) \\
& \left.-\frac{a_{12}\left(u_{2}-u_{2}^{*}\right)}{m_{1}+u_{1}}+\frac{a_{12} u_{2}^{*}\left(u_{1}-u_{1}^{*}\right)}{\left(m_{1}+u_{1}\right)\left(m_{1}+u_{1}^{*}\right)}\right], \\
\frac{\partial u_{2}}{\partial t}-d_{2} \Delta u_{2}=u_{2}[ & \int_{\Omega}^{t} \int_{-\infty}^{t} K_{1}(x, y, t-s) \\
& \times \frac{a_{21} m_{1}\left(u_{1}(s, y)-u_{1}^{*}\right)}{\left(m_{1}+u_{1}(s, y)\right)\left(m_{1}+u_{1}^{*}\right)} d s d y \\
& -a_{22}\left(u_{2}-u_{2}^{*}\right)-\frac{a_{23}\left(u_{3}-u_{3}^{*}\right)}{m_{2}+u_{2}} \\
& \left.+\frac{a_{23} u_{3}^{*}\left(u_{2}-u_{2}^{*}\right)}{\left.\left(m_{2}+u_{2}\right)\left(m_{2}+u_{2}^{*}\right)\right]}\right] \\
\frac{\partial u_{3}}{\partial t}-d_{3} \Delta u_{3}=u_{3} & {\left[\int_{\Omega}^{t} \int_{-\infty}^{t} K_{2}(x, y, t-s)\right.} \\
& \times \frac{a_{32} m_{2}\left(u_{2}(s, y)-u_{2}^{*}\right)}{\left(m_{2}+u_{2}(s, y)\right)\left(m_{2}+u_{2}^{*}\right)} d s d y \\
&
\end{aligned}
$$

Define

$$
\begin{aligned}
V(t)= & \alpha \int_{\Omega}\left(u_{1}-u_{1}^{*}-u_{1}^{*} \ln \frac{u_{1}}{u_{1}^{*}}\right) d x \\
& +\int_{\Omega}\left(u_{2}-u_{2}^{*}-u_{2}^{*} \ln \frac{u_{2}}{u_{2}^{*}}\right) d x \\
& +\beta \int_{\Omega}\left(u_{2}-u_{3}^{*}-u_{3}^{*} \ln \frac{u_{3}}{u_{3}^{*}}\right) d x
\end{aligned}
$$

where $\alpha$ and $\beta$ are positive constants to be determined. Calculating the derivatives $V(t)$ along the positive solution to the system (1)-(3) yields

$$
V^{\prime}(t)=\Phi_{1}(t)+\Phi_{2}(t)
$$

where

$$
\begin{gathered}
\Phi_{1}(t)=-\int_{\Omega}\left(\frac{\alpha d_{1} u_{1}^{*}}{u_{1}^{2}}\left|\nabla u_{1}\right|^{2}+\frac{d_{2} u_{2}^{*}}{u_{2}^{2}}\left|\nabla u_{2}\right|^{2}\right. \\
\left.+\frac{\beta d_{3} u_{3}^{*}}{u_{3}^{2}}\left|\nabla u_{3}\right|^{2}\right) d x,
\end{gathered}
$$




$$
\begin{aligned}
\Phi_{2}(t)= & -\int_{\Omega} \alpha\left[a_{11}-\frac{a_{12} u_{2}^{*}}{\left(m_{1}+u_{1}\right)\left(m_{1}+u_{1}^{*}\right)}\right]\left(u_{1}-u_{1}^{*}\right)^{2} d x \\
& -\int_{\Omega}\left[a_{22}-\frac{a_{23} u_{3}^{*}}{\left(m_{2}+u_{2}\right)\left(m_{2}+u_{2}^{*}\right)}\right]\left(u_{2}-u_{2}^{*}\right)^{2} d x \\
& -\int_{\Omega} a_{33} \beta\left(u_{3}-u_{3}^{*}\right)^{2} d x \\
& -\int_{\Omega} \frac{a_{23}\left(u_{3}-u_{3}^{*}\right)\left(u_{2}-u_{2}^{*}\right)}{m_{2}+u_{2}} d x \\
& -\int_{\Omega} \frac{\alpha a_{12}\left(u_{2}-u_{2}^{*}\right)\left(u_{1}-u_{1}^{*}\right)}{m_{1}+u_{1}} d x \\
& +\iint_{\Omega} \int_{-\infty}^{t} K_{1}(x, y, t-s) \\
& \times \frac{a_{21} m_{1}\left(u_{1}(s, y)-u_{1}^{*}\right)\left(u_{2}(t, x)-u_{2}^{*}\right)}{\left(m_{1}+u_{1}(s, y)\right)\left(m_{1}+u_{1}^{*}\right)} d s d y d x \\
& +\beta \iint_{\Omega} \int_{-\infty}^{t} K_{2}(x, y, t-s) \\
& \frac{a_{32} m_{2}\left(u_{2}(s, y)-u_{2}^{*}\right)\left(u_{3}(t, x)-u_{3}^{*}\right)}{\left(m_{2}+u_{2}(s, y)\right)\left(m_{2}+u_{2}^{*}\right)} d s d y d x .
\end{aligned}
$$

Applying the inequality $a b \leq \epsilon a^{2}+(1 / 4 \epsilon) b^{2}$, we derive from (25) that

$$
\begin{aligned}
\Phi_{2}(t) \leq & -\int_{\Omega} \alpha\left[a_{11}-\frac{a_{12} u_{2}^{*}}{\left(m_{1}+u_{1}\right)\left(m_{1}+u_{1}^{*}\right)}\right]\left(u_{1}-u_{1}^{*}\right)^{2} d x \\
& -\int_{\Omega} a_{33} \beta\left(u_{3}-u_{3}^{*}\right)^{2} d x \\
& -\int_{\Omega}\left[a_{22}-\frac{a_{23} u_{3}^{*}}{\left(m_{2}+u_{2}\right)\left(m_{2}+u_{2}^{*}\right)}\right]\left(u_{2}-u_{2}^{*}\right)^{2} d x \\
& +\int_{\Omega} \frac{\alpha a_{12}}{m_{1}+u_{1}}\left[\epsilon_{1}\left(u_{1}-u_{1}^{*}\right)^{2}+\frac{1}{4 \epsilon_{1}}\left(u_{2}-u_{2}^{*}\right)^{2}\right] d x \\
& +\int_{\Omega} \frac{a_{23}}{m_{2}+u_{2}}\left[\frac{1}{4 \epsilon_{2}}\left(u_{2}-u_{2}^{*}\right)^{2}+\epsilon_{2}\left(u_{3}-u_{3}^{*}\right)^{2}\right] d x \\
& +\iint_{\Omega} \int_{-\infty}^{t} K_{1}(x, y, t-s) \\
& \times \frac{a_{21} m_{1}}{\left(m_{1}+u_{1}(s, y)\right)\left(m_{1}+u_{1}^{*}\right)} \\
& \times\left[\epsilon_{3}\left(u_{1}(s, y)-u_{1}^{*}\right)^{2}\right. \\
& \left.+\frac{1}{4 \epsilon_{3}}\left(u_{2}(t, x)-u_{2}^{*}\right)^{2}\right] d s d y d x
\end{aligned}
$$

According to the property of the Kernel functions $K_{i}(x, y, t)$, $(i=1,2)$, we know that

$$
\begin{aligned}
\Phi_{2}(t) \leq & -\int_{\Omega}\left[\alpha a_{11}-\frac{\alpha a_{12} u_{2}^{*}}{m_{1}^{2}}-\frac{\alpha a_{12} \epsilon_{1}}{m_{1}}\right]\left(u_{1}-u_{1}^{*}\right)^{2} d x \\
& -\int_{\Omega}\left[a_{22}-\frac{a_{23} u_{3}^{*}}{m_{2}^{2}}-\frac{\alpha a_{12}}{4 m_{1} \epsilon_{1}}-\frac{a_{23}}{4 m_{2} \epsilon_{2}}\right. \\
& -\int_{\Omega}\left[a_{33} \beta-\frac{a_{21}}{4 m_{1} \epsilon_{3}}\right]\left(u_{2}-u_{2}^{*}\right)^{2} d x \\
+ & \frac{a_{21} \epsilon_{3}}{m_{1}} \iint_{\Omega} \int_{-\infty}^{t} K_{1}(x, y, t-s) \\
+ & \frac{a_{32} \beta}{4 m_{2} \epsilon_{4}} \iint_{\Omega} \int_{-\infty}^{t} K_{2}(x, y, t-s) \\
& \times\left(u_{1}(s, y)-u_{1}^{*}\right)^{2} d s d y d x
\end{aligned}
$$

Define a new Lyapunov functional as follows:

$$
\begin{aligned}
E(t)= & V(t) \\
& +\frac{a_{21} \epsilon_{3}}{m_{1}} \iint_{\Omega} \int_{0}^{+\infty} \int_{t-r}^{t} K_{1}(x, y, r) \\
& \times\left(u_{1}(s, y)-u_{1}^{*}\right)^{2} d s d r d y d x \\
+ & \frac{a_{32} \beta}{4 m_{2} \epsilon_{4}} \iint_{\Omega} \int_{0}^{+\infty} \int_{t-r}^{t} K_{2}(x, y, r) \\
& \times\left(u_{2}(s, y)-u_{2}^{*}\right)^{2} d s d r d y d x .
\end{aligned}
$$


It is derived from (27) and (28) that

$$
\begin{aligned}
E^{\prime}(t) \leq- & \int_{\Omega}\left(\frac{\alpha d_{1} u_{1}^{*}}{u_{1}^{2}}\left|\nabla u_{1}\right|^{2}+\frac{d_{2} u_{2}^{*}}{u_{2}^{2}}\left|\nabla u_{2}\right|^{2}\right. \\
& \left.+\frac{\beta d_{3} u_{3}^{*}}{u_{3}^{2}}\left|\nabla u_{3}\right|^{2}\right) d x \\
- & {\left[\alpha a_{11}-\frac{\alpha a_{12} u_{2}^{*}}{m_{1}^{2}}-\frac{\alpha a_{12} \epsilon_{1}}{m_{1}}\right.} \\
& \left.-\frac{a_{21} \epsilon_{3}}{m_{1}}\right] \int_{\Omega}\left(u_{1}-u_{1}^{*}\right)^{2} d x \\
- & {\left[a_{22}-\frac{a_{23} u_{3}^{*}}{m_{2}^{2}}-\frac{\alpha a_{12}}{4 m_{1} \epsilon_{1}}-\frac{a_{23}}{4 m_{2} \epsilon_{2}}-\frac{a_{21}}{4 m_{1} \epsilon_{3}}\right.} \\
- & {\left[a_{33} \beta-\frac{a_{32} \beta}{4 m_{2} \epsilon_{4}}\right] \int_{\Omega}\left(u_{2}-u_{2}^{*}\right)^{2} d x } \\
a_{2} & \left.\frac{\beta a_{32} \epsilon_{4}}{m_{2}}\right] \int_{\Omega}\left(u_{3}-u_{3}^{*}\right)^{2} d x .
\end{aligned}
$$

Since $\left(u_{1}, u_{2}, u_{3}\right)$ is the unique positive solution of system (1). Using Proposition 5, there exists a constant $C$ which does not depend on $x \in \bar{\Omega}$ or $t \geq 0$ such that $\left\|u_{i}(\cdot, t)\right\|_{\infty} \leq C(i=1,2,3)$ for $t \geq 0$. By Theorem $A_{2}$ in [24], we have

$$
\left\|u_{i}(\cdot, t)\right\|_{C^{2, \alpha}(\bar{\Omega})} \leq C, \quad \forall t \geq 1 .
$$

Assume that

$$
\begin{aligned}
& G_{1}: a_{11} m_{1}^{2}>a_{12} u_{2}^{*}, \\
& G_{2}: a_{22} m_{2}^{2}>a_{23} u_{3}^{*}+\frac{2 a_{23} a_{32}}{a_{33}}+\frac{2 a_{12} a_{21} m_{2}^{2}}{a_{11} m_{1}^{2}-a_{12} u_{2}^{*}},
\end{aligned}
$$

and denote

$$
\begin{aligned}
& l_{1}=\alpha a_{11}-\frac{\alpha a_{12} u_{2}^{*}}{m_{1}^{2}}-\frac{\alpha a_{12} \epsilon_{1}}{m_{1}}-\frac{a_{21} \epsilon_{3}}{m_{1}}, \\
& l_{2}=a_{22}-\frac{a_{23} u_{3}^{*}}{m_{2}^{2}}-\frac{\alpha a_{12}}{4 m_{1} \epsilon_{1}}-\frac{a_{23}}{4 m_{2} \epsilon_{2}}-\frac{a_{21}}{4 m_{1} \epsilon_{3}}-\frac{a_{32} \beta}{4 m_{2} \epsilon_{4}}, \\
& l_{3}=a_{33} \beta-\frac{a_{23} \epsilon_{2}}{m_{2}}-\frac{\beta a_{32} \epsilon_{4}}{m_{2}} .
\end{aligned}
$$

Then (29) is transformed into

$$
\begin{aligned}
E^{\prime}(t) \leq- & \int_{\Omega}\left(\frac{\alpha d_{1} u_{1}^{*}}{u_{1}^{2}}\left|\nabla u_{1}\right|^{2}+\frac{d_{2} u_{2}^{*}}{u_{2}^{2}}\left|\nabla u_{2}\right|^{2}\right. \\
& \left.+\frac{\beta d_{3} u_{3}^{*}}{u_{3}^{2}}\left|\nabla u_{3}\right|^{2}\right) d x \\
& -\sum_{i=1}^{3} l_{i} \int_{\Omega}\left(u_{i}-u_{i}^{*}\right)^{2} d x .
\end{aligned}
$$

If we choose

$$
\begin{aligned}
\alpha=\frac{a_{21}}{a_{12}}, \quad \beta & =\frac{a_{23}}{a_{32}}, \quad \epsilon_{1}=\epsilon_{3}=\frac{a_{11} m_{1}^{2}-a_{12} u_{2}^{*}}{4 m_{1} a_{12}}, \\
\epsilon_{2} & =\epsilon_{4}=\frac{m_{2} a_{33}}{4 a_{32}},
\end{aligned}
$$

then $l_{i}>0(i=1,2,3)$. Therefore, we have

$$
E^{\prime}(t) \leq-\sum_{i=1}^{3} l_{i} \int_{\Omega}\left(u_{i}-u_{i}^{*}\right)^{2} d x .
$$

From Proposition 6 we can see that the solution of system (1) and (3) is bounded, and so are the derivatives of $\left(u_{i}-u_{i}^{*}\right)$ $(i=1,2,3)$ by the equations in (1). Applying Lemma 1, we obtain that

$$
\lim _{t \rightarrow \infty} \int_{\Omega}\left(u_{i}-u_{i}^{*}\right) d x=0, \quad(i=1,2,3) .
$$

Recomputing $E^{\prime}(t)$ gives

$$
\begin{gathered}
E^{\prime}(t) \leq-\int_{\Omega}\left(\frac{\alpha d_{1} u_{1}^{*}}{u_{1}^{2}}\left|\nabla u_{1}\right|^{2}+\frac{d_{2} u_{2}^{*}}{u_{2}^{2}}\left|\nabla u_{2}\right|^{2}\right. \\
\left.+\frac{\beta d_{3} u_{3}^{*}}{u_{3}^{2}}\left|\nabla u_{3}\right|^{2}\right) d x \\
\leq-c \int_{\Omega}\left(\left|\nabla u_{1}\right|^{2}+\left|\nabla u_{2}\right|^{2}+\left|\nabla u_{3}\right|^{2}\right) d x=-g(t),
\end{gathered}
$$

where $c=\min \left\{\alpha d_{1} u_{1}^{*} / M_{1}^{2}, d_{2} u_{2}^{*} / M_{2}^{2}, \beta d_{3} u_{3}^{*} / M_{3}^{2}\right\}$. Using (30) and (1), we obtain that the derivative of $g(t)$ is bounded in $[1,+\infty)$. From Lemma 1 , we conclude that $g(t) \rightarrow 0$ as $t \rightarrow$ $\infty$. Therefore

$$
\lim _{t \rightarrow \infty} \int_{\Omega}\left(\left|\nabla u_{1}\right|^{2}+\left|\nabla u_{2}\right|^{2}+\left|\nabla u_{3}\right|^{2}\right) d x=0 .
$$

Applying the Poincaré inequality

$$
\int_{\Omega} \lambda|u-\bar{u}|^{2} d x \leq \int_{\Omega}|\nabla u|^{2} d x
$$

leads to

$$
\lim _{t \rightarrow \infty} \int_{\Omega}\left(u_{i}-\bar{u}_{i}\right)^{2} d x=0, \quad(i=1,2,3)
$$

where $\bar{u}_{i}=(1 /|\Omega|) \int_{\Omega} u_{i} d x$ and $\lambda$ is the smallest positive eigenvalue of $-\Delta$ with the homogeneous Neumann condition. Therefore,

$$
\begin{aligned}
|\Omega|\left(\bar{u}_{1}(t)-u_{1}^{*}\right)^{2} & =\int_{\Omega}\left(\bar{u}_{1}(t)-u_{1}^{*}\right)^{2} d x \\
& =\int_{\Omega}\left(\bar{u}_{1}(t)-u_{1}(t, x)+u_{1}(t, x)-u_{1}^{*}\right)^{2} d x \\
& \leq 2 \int_{\Omega}\left(\bar{u}_{1}(t)-u_{1}(t, x)\right)^{2} d x \\
& +2 \int_{\Omega}\left(u_{1}(t, x)-u_{1}^{*}\right)^{2} d x .
\end{aligned}
$$


So we have $\bar{u}_{1}(t) \rightarrow u_{1}^{*}$ as $t \rightarrow \infty$. Similarly, $\bar{u}_{2}(t) \rightarrow u_{2}^{*}$ and $\bar{u}_{3}(t) \rightarrow u_{3}^{*}$ as $t \rightarrow \infty$. According to (30), there exists a subsequence $t_{m}$, and non-negative functions $w_{i} \in C^{2}(\bar{\Omega})$, such that

$$
\lim _{m \rightarrow \infty}\left\|u_{i}\left(\cdot, t_{m}\right)-w_{i}(\cdot)\right\|_{C^{2}(\bar{\Omega})}=0 \quad(i=1,2,3) .
$$

Applying (40) and noting that $\bar{u}_{i}(t) \rightarrow u_{i}^{*}$, we then have $w_{i}=$ $u_{i}^{*},(i=1,2,3)$. That is,

$$
\lim _{m \rightarrow \infty}\left\|u_{i}\left(\cdot, t_{m}\right)-u_{i}^{*}\right\|_{C^{2}(\bar{\Omega})}=0 \quad(i=1,2,3) .
$$

Furthermore, the local stability of $\mathbf{E}^{*}$ combining with (43) gives the following global stability.

Theorem 9. Assume that

$$
H_{5}: \frac{a_{1}\left(a_{21}-a_{2}\right)}{m_{1} a_{2}}>a_{11}>\frac{a_{12} v_{2}^{*}}{m_{1}^{2}},
$$

$H_{4}$ and $G_{4}$ hold, and then the semi-trivial steady state $E_{2}$ of system (1)-(3) with non-trivial initial functions is globally asymptotically stable.

Proof. It is obvious that system (1)-(3) always has two nonnegative equilibria $\left(u_{1}, u_{2}, u_{3}\right)$ as follows: $E_{0}=(0,0,0)$ and $E_{1}=\left(a_{1} / a_{11}, 0,0\right)$. If $H_{1}$ and $H_{4}$ are satisfied, system (1) has the other semitrivial solution denoted by $E_{2}\left(v_{1}^{*}, v_{2}^{*}, 0\right)$, where

$$
H_{4}: a_{3} m_{2}>\left(a_{32}-a_{3}\right) v_{2}^{*} \text {. }
$$

We consider the stability of $E_{2}$ under condition $H_{1}$ and $H_{4}$. Equation (1) can be rewritten as

$$
\begin{aligned}
\frac{\partial u_{1}}{\partial t}-d_{1} \Delta u_{1}=u_{1}[ & -a_{11}\left(u_{1}-v_{1}^{*}\right)-\frac{a_{12}\left(u_{2}-v_{2}^{*}\right)}{m_{1}+u_{1}} \\
& \left.+\frac{a_{12} v_{2}^{*}\left(u_{1}-v_{1}^{*}\right)}{\left(m_{1}+u_{1}\right)\left(m_{1}+v_{1}^{*}\right)}\right],
\end{aligned}
$$

$$
\begin{aligned}
\frac{\partial u_{2}}{\partial t}-d_{2} \Delta u_{2} & \\
=u_{2}\left[\int_{\Omega} \int_{-\infty}^{t} K_{1}(x, y, t-s)\right. & \\
& \times \frac{a_{21} m_{1}\left(u_{1}(s, y)-v_{1}^{*}\right)}{\left(m_{1}+u_{1}(s, y)\right)\left(m_{1}+v_{1}^{*}\right)} d s d y \\
& \left.-a_{22}\left(u_{2}-v_{2}^{*}\right)-\frac{a_{23} u_{3}}{m_{2}+u_{2}}\right],
\end{aligned}
$$

$$
\begin{aligned}
& \frac{\partial u_{3}}{\partial t}-d_{3} \Delta u_{3} \\
&=u_{3}\left[\int_{\Omega} \int_{-\infty}^{t} K_{2}(x, y, t-s)\right. \\
& \\
& \quad \times \frac{a_{32} m_{2}\left(u_{2}(s, y)-v_{2}^{*}\right)}{\left(m_{2}+u_{2}(s, y)\right)\left(m_{2}+v_{2}^{*}\right)} d s d y \\
&\left.\quad-a_{33} u_{3}\right] .
\end{aligned}
$$

Similar to the argument of Theorem 8 , we have $u_{1}(x, t) \rightarrow v_{1}^{*}$ and $u_{2}(x, t) \rightarrow v_{2}^{*}$ as $t \rightarrow \infty$ uniformly on $\bar{\Omega}$ provided that the following additional condition holds:

$$
\begin{aligned}
& G_{3}: \quad a_{11} m_{1}^{2}>a_{12} v_{2}^{*}, \\
& G_{4}: \quad a_{22} m_{2}^{2}>\frac{a_{23} a_{32}}{a_{33}}+\frac{2 a_{12} a_{21} m_{2}^{2}}{a_{11} m_{1}^{2}-a_{12} v_{2}^{*}} .
\end{aligned}
$$

Next, we consider the asymptotic behavior of $u_{3}(t, x)$. Let

$$
\theta=\frac{a_{3}-\left(a_{32}-a_{3}\right) v_{2}^{*}}{2 \delta}, \quad \delta=\left|a_{32}-a_{3}\right|
$$

Then there exists $t_{1}>0$ such that

$$
v_{2}^{*}-\theta<u_{2}(t, x)<v_{2}^{*}+\theta, \quad \forall t>t_{1}, x \in \bar{\Omega} .
$$

Consider the following two systems:

$$
\begin{array}{r}
w_{3 t}-d_{3} \Delta w_{3}=w_{3}\left[-a_{3}+\frac{a_{32}\left(v_{2}^{*}+\theta\right)}{m_{2}+v_{2}^{*}+\theta}-a_{33} w_{3}\right], \\
(t, x) \in\left[t_{1},+\infty\right) \times \Omega,
\end{array}
$$

$$
\begin{aligned}
& \frac{\partial w_{3}}{\partial \nu}=0, \quad(t, x) \in\left[t_{1},+\infty\right) \times \partial \Omega, \\
& w_{3}=u_{3}, \quad(t, x) \in\left(-\infty, t_{1}\right] \times \Omega, \\
& W_{3 t}-d_{3} \Delta W_{3}=W_{3}\left[-a_{3}+\frac{a_{32}\left(v_{2}^{*}-\theta\right)}{m_{2}+v_{2}^{*}-\theta}-a_{33} v_{3}\right], \\
& (t, x) \in\left[t_{1},+\infty\right) \times \Omega,
\end{aligned}
$$

$$
\begin{aligned}
& \frac{\partial W_{3}}{\partial \nu}=0, \quad(t, x) \in\left[t_{1},+\infty\right) \times \partial \Omega, \\
& W_{3}=u_{3}, \quad(t, x) \in\left(-\infty, t_{1}\right] \times \Omega .
\end{aligned}
$$

Combining comparison principle with (50), we obtain that

$$
W_{3}(t, x) \leq u_{3}(t, x) \leq w_{3}(t, x), \quad \forall t>t_{1}, x \in \bar{\Omega} .
$$

By Lemma 4, we obtain

$$
\begin{aligned}
0=\lim _{t \rightarrow \infty} W_{3}(t, x) & \leq \inf u_{3}(t, x) \leq \sup u_{3}(t, x) \\
& \leq \lim _{t \rightarrow \infty} w_{3}(t, x)=0, \quad \forall x \in \bar{\Omega},
\end{aligned}
$$

which implies that $\lim _{t \rightarrow \infty} u_{3}(t, x)=0$ uniformly on $\bar{\Omega}$.

Theorem 10. Suppose that $G_{5}$ and $G_{6}$ hold, and then the semitrivial steady state $E_{1}$ of system (1)-(3) with non-trivial initial functions is globally asymptotically stable. 
Proof. We study the stability of the semi-trivial solution $E_{1}=$ $\left(\widetilde{u}_{1}, 0,0\right)$. Similarly, the equations in (1) can be written as

$$
\begin{aligned}
& \frac{\partial u_{1}}{\partial t}-d_{1} \Delta u_{1}=u_{1}\left[-a_{11}\left(u_{1}-\tilde{u}_{1}\right)-\frac{a_{12} u_{2}}{m_{1}+u_{1}}\right] \\
& \frac{\partial u_{2}}{\partial t}-d_{2} \Delta u_{2} \\
& =u_{2}\left[-a_{2}+\int_{\Omega} \int_{-\infty}^{t} K_{1}(x, y, t-s)\right. \\
& \times \frac{a_{21} m_{1}\left(u_{1}(s, y)-\tilde{u}_{1}\right)}{\left(m_{1}+u_{1}(s, y)\right)\left(m_{1}+\tilde{u}_{1}\right)} d s d y \\
& \left.+\frac{a_{21} \tilde{u}_{1}}{m_{1}+\tilde{u}_{1}}-a_{22} u_{2}-\frac{a_{23} u_{3}}{m_{2}+u_{2}}\right], \\
& \frac{\partial u_{3}}{\partial t}-d_{3} \Delta u_{3} \\
& =u_{3}\left[-a_{3}+\int_{\Omega}^{t} \int_{-\infty} K_{2}(x, y, t-s)\right. \\
& \left.\times \frac{a_{32} u_{2}(s, y)}{m_{2}+u_{2}(s, y)} d s d y-a_{33} u_{3}\right] .
\end{aligned}
$$

Define

$$
\begin{aligned}
V(t)= & \alpha \int_{\Omega}\left[u_{1}-\tilde{u}_{1}-\tilde{u}_{1} \log \frac{u_{1}}{\tilde{u}_{1}}\right] d x+\int_{\Omega} u_{2} d x \\
& +\beta \int_{\Omega} u_{3} d x
\end{aligned}
$$

Calculating the derivative of $V(t)$ along $E_{1}$, we get from (54) that

$$
\begin{aligned}
& V^{\prime}(t) \leq-\int_{\Omega}\left[\alpha\left(a_{11}-\frac{a_{12} \epsilon_{1}}{m_{1}}\right)\right]\left(u_{1}-\tilde{u}_{1}\right)^{2} d x \\
&+\int_{\Omega}\left[a_{22}-\frac{\alpha a_{12}}{4 m_{1} \epsilon_{1}}-\frac{a_{23}}{4 \epsilon_{2} m_{2}}-\frac{a_{21}}{4 m_{1} \epsilon_{3}}\right] u_{2}^{2} d x \\
&+\int_{\Omega}\left[\beta a_{33}-\frac{a_{23} \epsilon_{2}}{m_{2}}\right] u_{3}^{2} d x \\
&-\int_{\Omega}\left[\left(a_{2}-\frac{a_{21} \tilde{u}_{1}}{m_{1}+\widetilde{u}_{1}}\right) u_{2}+a_{3} u_{3}\right] d x \\
&+\frac{a_{21} \epsilon_{3}}{m_{1}} \int_{\Omega} \int_{-\infty}^{t} K_{1}(x, y, t-s) \\
&+\frac{a_{32} \beta}{4 \epsilon_{4} m_{2}} \iint_{\Omega} \int_{-\infty}^{t} K_{2}(x, y, t-s) \\
& \times\left(u_{1}(s, y)-\tilde{u}_{1}\right)^{2} d s d y d x
\end{aligned}
$$

Define

$$
\begin{aligned}
E(t)= & V(t) \\
& +\frac{a_{21} \epsilon_{3}}{m_{1}} \iint_{\Omega} \int_{0}^{+\infty} \int_{t-r}^{t} K_{1}(x, y, r) \\
& \times\left(u_{1}(s, y)-u_{1}^{*}\right)^{2} d s d r d y d x \\
& +\frac{a_{32} \beta}{4 \epsilon_{4} m_{2}} \iint_{\Omega} \int_{0}^{+\infty} \int_{t-r}^{t} K_{2}(x, y, r) \\
& \times\left(u_{2}(s, y)-u_{2}^{*}\right)^{2} d s d r d y d x
\end{aligned}
$$

It is easy to see that

$$
\begin{aligned}
E^{\prime}(t) \leq & -\alpha d_{1} \tilde{u}_{1} \int_{\Omega} \frac{\left|\nabla u_{1}\right|^{2}}{u_{1}^{2}} d x \\
& -\int_{\Omega}\left[\alpha\left(a_{11}-\frac{a_{12} \epsilon_{1}}{m_{1}}\right)-\frac{a_{21} \epsilon_{3}}{m_{1}}\right]\left(u_{1}-\tilde{u}_{1}\right)^{2} d x \\
& +\int_{\Omega}\left[a_{22}-\frac{\alpha a_{12}}{4 m_{1} \epsilon_{1}}-\frac{a_{23}}{4 m_{2} \epsilon_{2}}-\frac{a_{21}}{4 m_{1} \epsilon_{3}}\right. \\
& +\int_{\Omega}\left[\beta a_{33}-\frac{a_{23} \epsilon_{2}}{m_{2}}-\frac{a_{32} \beta \epsilon_{4}}{m_{2}}\right] u_{3}^{2} d x \\
& -\int_{\Omega}\left[\left(a_{2}-\frac{a_{21} \tilde{u}_{1}}{m_{1}+\widetilde{u}_{1}}\right) u_{2}-a_{3} \beta u_{3}\right] d x .
\end{aligned}
$$

Assume that

$$
\begin{aligned}
& G_{5}: \quad a_{1}\left(a_{21}-a_{2}\right)<m_{1} a_{2} a_{11}, \\
& G_{6}: a_{22} m_{2}^{2}>\frac{a_{23} a_{32}}{a_{33}}+\frac{2 a_{12} a_{21} m_{2}^{2}}{a_{11} m_{1}^{2}} .
\end{aligned}
$$

Let

$$
\begin{aligned}
& l_{1}=\alpha a_{11}-\frac{\alpha a_{12} \epsilon_{1}}{m_{1}}-\frac{a_{21} \epsilon_{3}}{m_{1}} \\
& l_{2}=a_{22}-\frac{\alpha a_{12}}{4 m_{1} \epsilon_{1}}-\frac{a_{23}}{4 m_{2} \epsilon_{2}}-\frac{a_{21}}{4 m_{1} \epsilon_{3}}-\frac{a_{32} \beta}{4 m_{2} \epsilon_{4}}, \\
& l_{3}=a_{33} \beta-\frac{a_{23} \epsilon_{2}}{m_{2}}-\frac{\beta a_{32} \epsilon_{4}}{m_{2}}
\end{aligned}
$$

Choose

$$
\begin{gathered}
\alpha=\frac{a_{21}}{a_{12}}, \quad \beta=\frac{a_{23}}{a_{32}}, \quad \epsilon_{1}=\epsilon_{3}=\frac{a_{11} m_{1}}{4 a_{12}}, \\
\epsilon_{2}=\epsilon_{4}=\frac{m_{2} a_{33}}{4 a_{32}} .
\end{gathered}
$$

Then we get $l_{i}>0(i=1,2,3)$. Therefore we have

$$
\lim _{t \rightarrow \infty} u_{1}(t, x)=\widetilde{u}_{1}
$$

uniformly on $\bar{\Omega}$. 


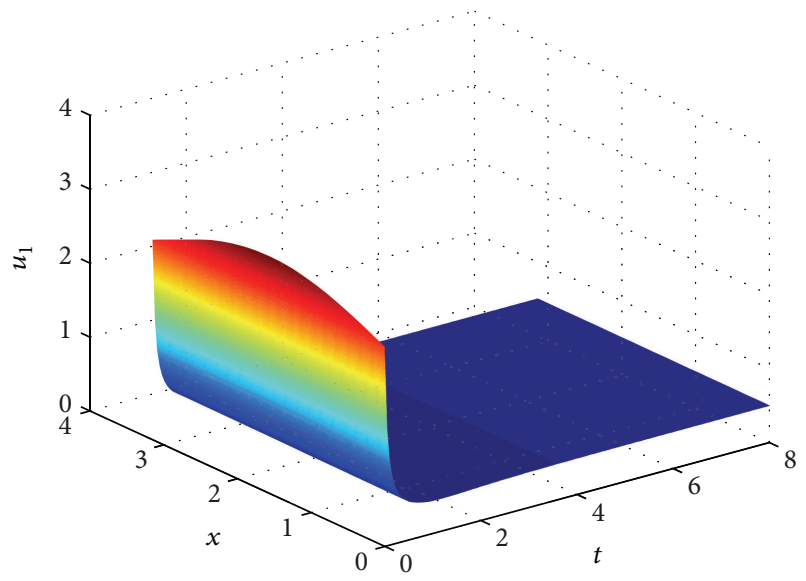

(a)

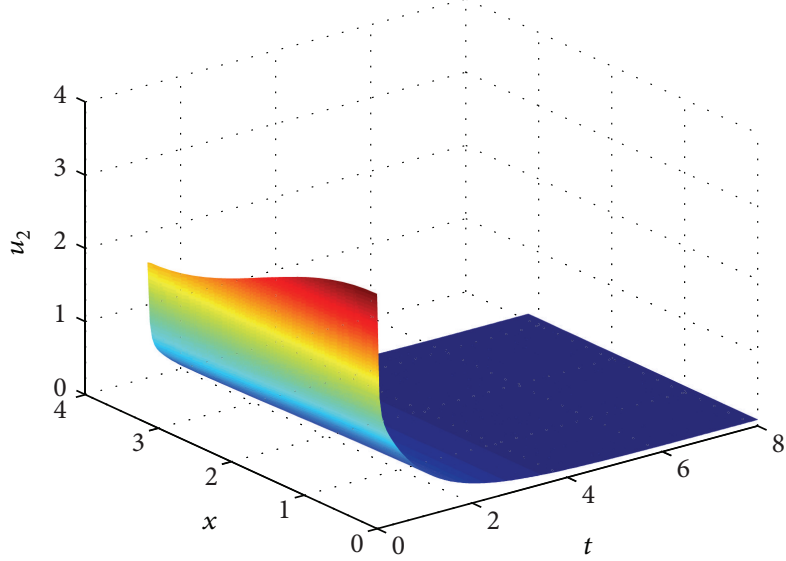

(b)

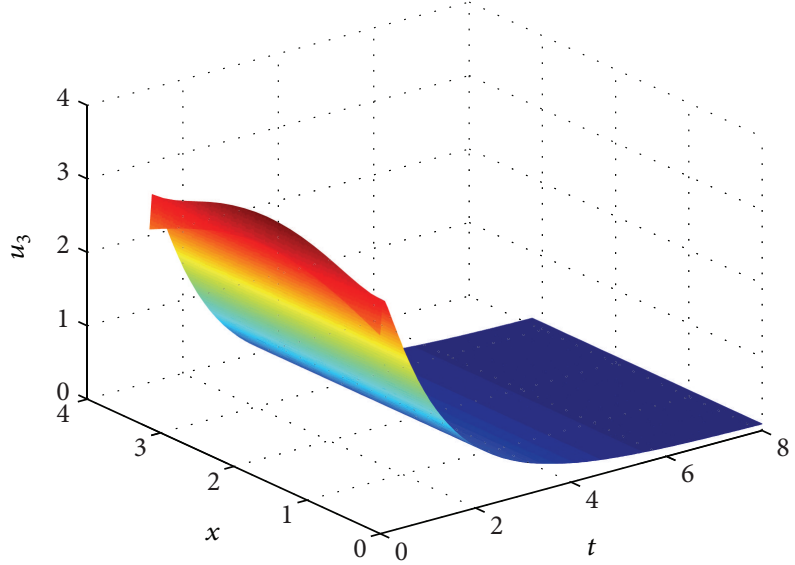

(c)

FIGURE 2: Global stability of the positive equilibrium $E^{*}$ with an initial condition $\left(\phi_{1}, \phi_{2}, \phi_{3}\right)=(2.7+0.5 \sin x, 2.7+0.5 \cos x, 2.7+0.5 \sin x)$.

Next, we consider the asymptotic behavior of $u_{2}(t, x)$ and $u_{3}(t, x)$. For any $T>0$, integrating $(57)$ over $[0, T]$ yields

$$
\begin{aligned}
E(T) & +\alpha d_{1}\left\|\left|\frac{\nabla u_{1}}{u_{1}}\right|\right\|_{2}^{2}+l_{1}\left\|\left|u_{1}-\widetilde{u}_{1}\right|\right\|_{2}^{2} \\
& +l_{2}\left\|\left|u_{2}\right|\right\|_{2}^{2}+l_{3}\left\|\left|u_{3}\right|\right\|_{2}^{2} \leq E(0),
\end{aligned}
$$

where $\left\|\left|u_{i}\right|\right\|_{2}^{2}=\int_{0}^{T} \int_{\Omega} u_{i}^{2} d x d t$. It implies that $\left\|\left|u_{i}\right|\right\|_{2} \leq C_{i}(i=$ $2,3)$ for the constant $C_{i}$ which is independent of $T$. Now we consider the boundedness of $\left\|\left|\nabla u_{2}\right|\right\|_{2}$ and $\left\|\left|\nabla u_{3}\right|\right\|_{2}$. From the Green's identity, we obtain

$$
\begin{aligned}
& d_{2} \int_{0}^{T} \int_{\Omega}\left|\nabla u_{2}(t, x)\right|^{2} d x d t \\
& \quad=-d_{2} \int_{0}^{T} \int_{\Omega} u_{2}(t, x) \Delta u_{2}(t, x) d x d t \\
& \quad=-\int_{0}^{T} \int_{\Omega} u_{2} \frac{\partial u_{2}}{\partial t} d x d t-a_{2} \int_{0}^{T} \int_{\Omega} u_{2}^{2} d x d t
\end{aligned}
$$

$$
\begin{array}{r}
-a_{22} \int_{0}^{T} \int_{\Omega} u_{2}^{3} d x d t-a_{23} \int_{0}^{T} \int_{\Omega} \frac{u_{2}^{2} u_{3}}{m_{2}+u_{3}} d x d t \\
+a_{21} \int_{0}^{T} \iint_{\Omega} \int_{-\infty}^{t} \frac{u_{1}(s, y) u_{2}^{2}(t, x)}{m_{1}+u_{1}(s, y)} \\
\times K_{1}(x, y, t-s) d s d y d x d t
\end{array}
$$

Note that

$$
\begin{aligned}
& \int_{0}^{T} \int_{\Omega} u_{2}(t, x) \frac{\partial u_{2}(t, x)}{\partial t} d x d t \\
& \quad=\int_{0}^{T} \int_{\Omega} \frac{1}{2} \frac{\partial u_{2}^{2}}{\partial t} d t d x \\
& \quad=\frac{1}{2} \int_{\Omega} u_{2}^{2}(T, x) d x-\frac{1}{2} \int_{\Omega} u_{2}^{2}(0, x) d x \leq M_{2}^{2}|\Omega|, \\
& \int_{0}^{T} \int_{\Omega} u_{2}^{2} u_{3} d x d t \leq M_{3} \int_{0}^{T} \int_{\Omega} u_{2}^{2} d x d t,
\end{aligned}
$$




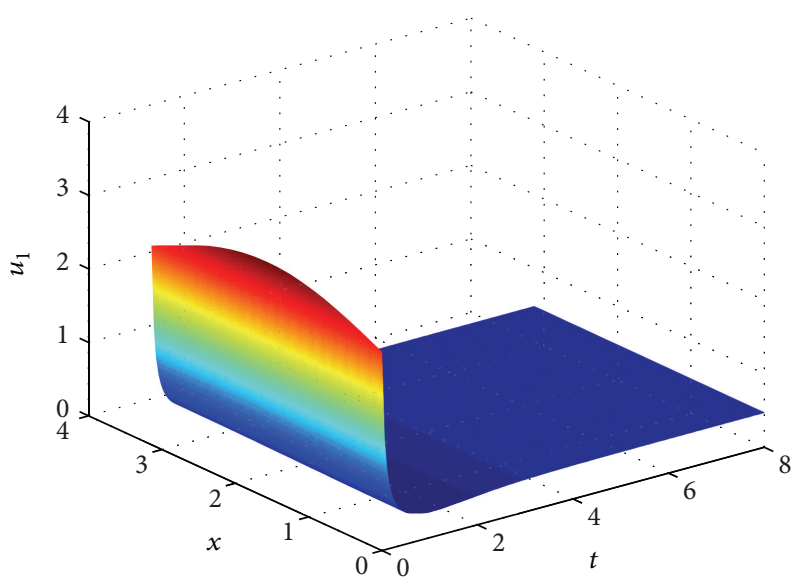

(a)

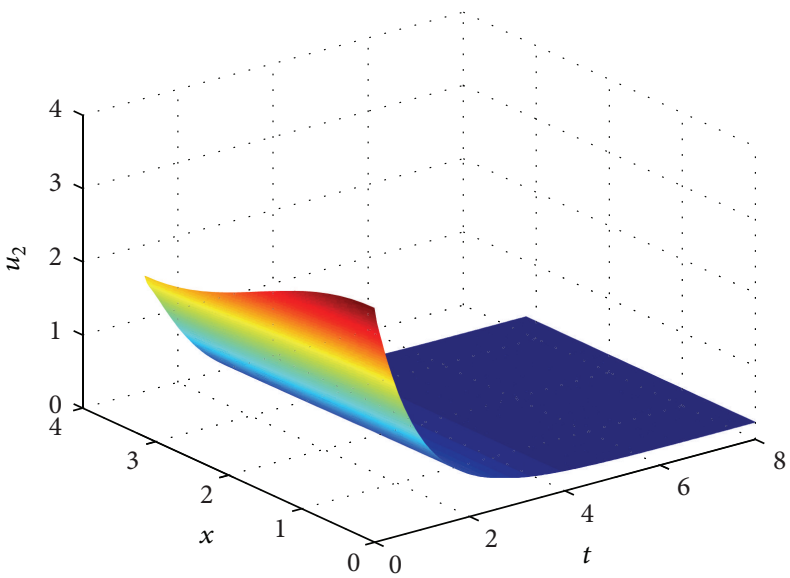

(b)

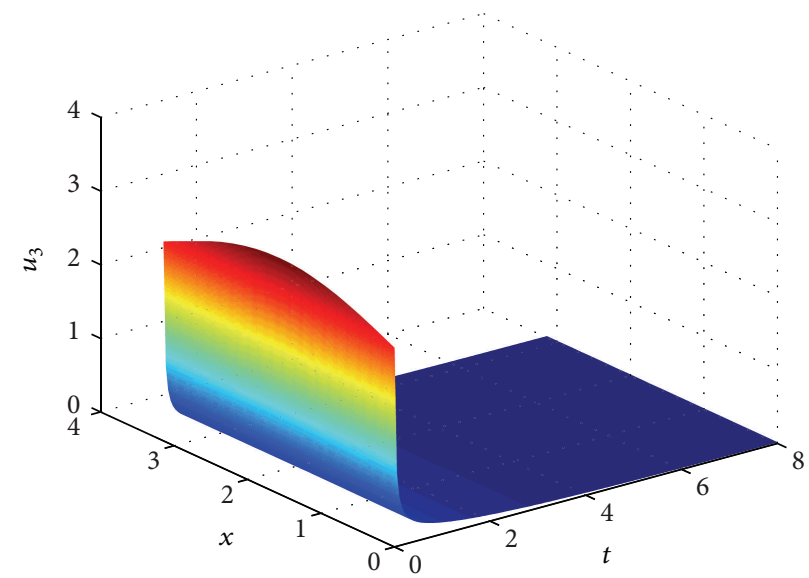

(c)

Figure 3: Global stability of the positive equilibrium $E_{2}$ with an initial condition $\left(\phi_{1}, \phi_{2}, \phi_{3}\right)=(2.7+0.5 \sin x, 2.7+0.5 \cos x, 2.7+0.5 \sin x)$.

$$
\begin{aligned}
& \int_{0}^{T} \int_{\Omega} u_{2}^{3} d x d t \leq M_{2} \int_{0}^{T} \int_{\Omega} u_{2}^{2} d x d t \\
& \int_{0}^{T} \iint_{\Omega} \int_{-\infty}^{t} \frac{u_{1}(s, y) u_{2}^{2}(t, x)}{m_{1}+u_{1}(s, y)} K_{1}(x, y, t-s) d s d y d x d t \\
& \quad \leq \int_{0}^{T} \int_{\Omega} u_{2}^{2}(t, x) d x d t .
\end{aligned}
$$

Thus, we get $\left\|\left|\nabla u_{2}\right|\right\|_{2} \leq C_{4}$. In a similar way, we have $\left\|\left|\nabla u_{3}\right|\right\|_{2} \leq C_{5}$. Here $C_{4}$ and $C_{5}$ are independent of $T$.

It is easy to see that $u_{2}(t, x), u_{3}(t, x) \in L^{2}((0, \infty)$; $\left.W^{1,2}(\Omega)\right)$. These imply that

$$
\lim _{t \rightarrow \infty}\left\|u_{i}(\cdot, t)\right\|_{W^{1,2}(\Omega)}=0, \quad i=2,3
$$

From the Sobolev compact embedding theorem, we know

$$
\lim _{t \rightarrow \infty}\left\|u_{i}(\cdot, t)\right\|_{C(\bar{\Omega})}=0, \quad i=2,3
$$

In the end, we show that the trivial solution $E_{0}$ is an unstable equilibrium. Similarly to the local stability to $E^{*}$, we can get the characteristic equation of $E_{0}$ as

$$
\left(\lambda+\mu_{i} D_{1}-a_{1}\right)\left(\lambda+\mu_{i} D_{2}+a_{2}\right)\left(\lambda+\mu_{i} D_{3}+a_{3}\right)=0 .
$$

If $i=1$, then $\mu_{1}=0$. It is easy to see that this equation admits a positive solution $\lambda=a_{1}$. According to Theorem 5.1 in [23], we have the following result.

Theorem 11. The trivial equilibrium $E_{0}$ is an unstable equilibrium of system (1)-(3).

\section{Numerical Illustrations}

In this section, we perform numerical simulations to illustrate the theoretical results given in Section 3. 


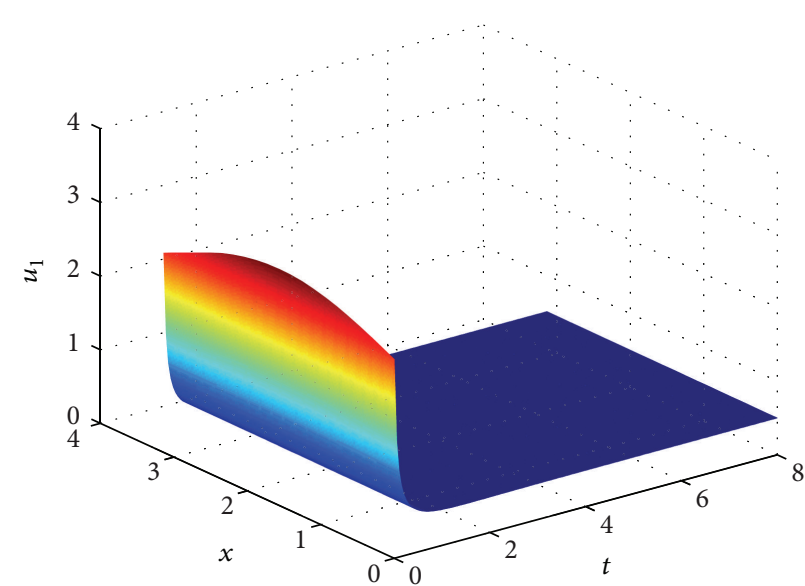

(a)

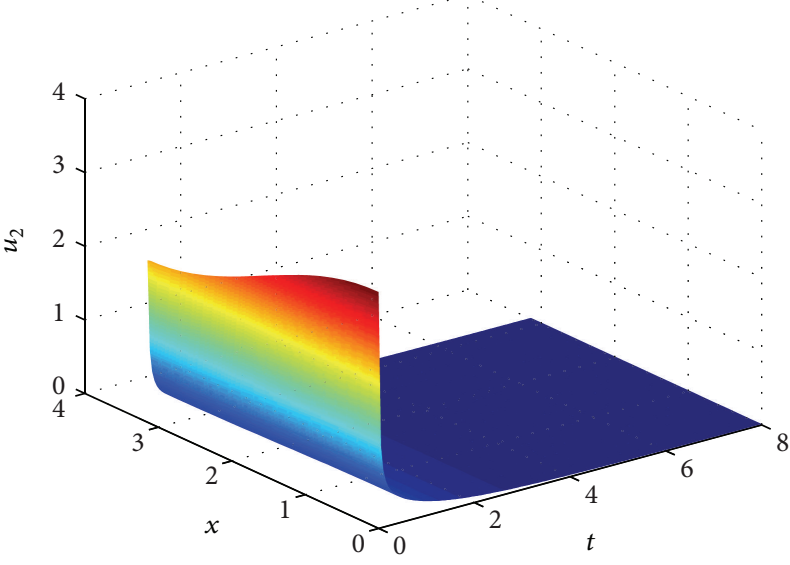

(b)

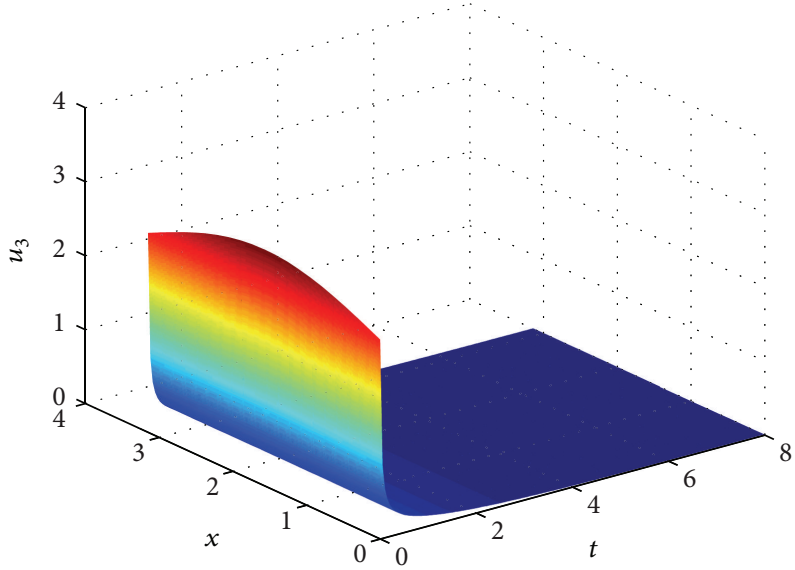

(c)

Figure 4: Global stability of the positive equilibrium $E_{1}$ with an initial condition $\left(\phi_{1}, \phi_{2}, \phi_{3}\right)=(2.7+0.5 \sin x, 2.7+0.5 \cos x, 2.7+0.5 \sin x)$.

In the following, we always take $\Omega=[0, \pi], K_{i}(x, y, t)=$ $G_{i}(x, y, t) k_{i}(t)$, where $k_{i}(t)=\left(1 / \tau^{*}\right) e^{-t / \tau^{*}}(i=1,2)$ and

$$
\begin{aligned}
& G_{1}(x, y, t)=\frac{1}{\pi}+\frac{2}{\pi} \sum_{n=1}^{\infty} e^{-d_{2} n^{2} t} \cos n x \cos n y, \\
& G_{2}(x, y, t)=\frac{1}{\pi}+\frac{2}{\pi} \sum_{n=1}^{\infty} e^{-d_{3} n^{2} t} \cos n x \cos n y .
\end{aligned}
$$

However, it is difficult for us to simulate our results directly because of the nonlocal term. Similar to [25], the equations in (1) can be rewritten as follows:

$$
\begin{aligned}
& \frac{\partial u_{1}}{\partial t}-d_{1} \Delta u_{1}=u_{1}\left(a_{1}-a_{11} u_{1}-\frac{a_{12} u_{2}}{m_{1}+u_{1}}\right), \\
& \frac{\partial u_{2}}{\partial t}-d_{2} \Delta u_{2}=u_{2}\left(-a_{2}+a_{21} v_{1}-a_{22} u_{2}-\frac{a_{23} u_{3}}{m_{2}+u_{3}}\right), \\
& \frac{\partial u_{3}}{\partial t}-d_{3} \Delta u_{3}=u_{3}\left(-a_{3}+a_{32} v_{2}-a_{33} u_{3}\right),
\end{aligned}
$$

$$
\begin{aligned}
& \frac{\partial v_{1}}{\partial t}-d_{2} \Delta v_{1}=\frac{1}{\tau^{*}}\left(\frac{u_{1}}{m_{1}+u_{1}}-v_{1}\right), \\
& \frac{\partial v_{2}}{\partial t}-d_{3} \Delta v_{2}=\frac{1}{\tau^{*}}\left(\frac{u_{2}}{m_{2}+u_{2}}-v_{2}\right),
\end{aligned}
$$

where

$$
\begin{array}{r}
v_{i}=\int_{0}^{\pi} \int_{-\infty}^{t} G_{i}(x, y, t-s) \frac{1}{\tau^{*}} e^{-(t-s) / \tau^{*}} \frac{u_{i}(s, y)}{m_{i}+u_{i}(s, y)} d y d s, \\
i=1,2 .
\end{array}
$$

Each component is considered with homogeneous Neumann boundary conditions, and the initial condition of $v_{i}$ is

$$
\begin{array}{r}
v_{i}(0, x)=\int_{0}^{\pi} \int_{-\infty}^{0} G_{i}(x, y,-s) \frac{1}{\tau^{*}} e^{s / \tau^{*}} \frac{u_{i}(s, y)}{m_{i}+u_{i}(s, y)} d y d s \\
i=1,2 .
\end{array}
$$


In the following examples, we fix some coefficients and assume that $d_{1}=d_{2}=d_{3}=1, a_{1}=3, a_{12}=1, m_{1}=m_{2}=$ $1, a_{2}=1, a_{22}=12, a_{23}=1, a_{33}=12$, and $\tau^{*}=1$. The asymptotic behaviors of system (1)-(3) are shown by choosing different coefficients $a_{11}, a_{21}, a_{3}$, and $a_{32}$.

Example 12. Let $a_{11}=53 / 9, a_{21}=81 / 13, a_{3}=1 / 13$ and $a_{32}=14$. Then it is easy to see that the system admits a unique positive equilibrium $E^{*}(1 / 2,1 / 12,1 / 12)$. By Theorem 8 , we see that the positive solution $\left(u_{1}(t, x), u_{2}(t, x), u_{3}(t, x)\right)$ of system (1)-(3) converges to $E^{*}$ as $t \rightarrow \infty$. See Figure 2.

Example 13. Let $a_{11}=6, a_{21}=12, a_{3}=1 / 4$ and $a_{32}=1$. Clearly, $H_{2}$ does not hold. Hence, the positive steady state is not feasible. System (1) admits two semitrivial steady state $E_{2}(0.4732,0.2372,0)$ and $E_{1}(1 / 2,0,0)$. According to Theorem 9, we know that the positive solution $\left(u_{1}(t, x), u_{2}(t, x), u_{3}(t, x)\right)$ of system (1)-(3) converges to $E_{2}$ as $t \rightarrow \infty$. See Figure 3 .

Example 14. Let $a_{11}=6, a_{21}=2, a_{3}=1 / 4$ and $a_{32}=1$. Clearly, $H_{1}$ does not hold. Hence, $E^{*}$ and $E_{2}$ are not feasible. System (1) has a unique semi-trivial steady state $E_{1}(1 / 2,0,0)$. According to Theorem 10 we know that the positive solution $\left(u_{1}(t, x), u_{2}(t, x), u_{3}(t, x)\right)$ of system (1)-(3) converges to $E_{1}$ as $t \rightarrow \infty$. See Figure 4 .

\section{Discussion}

In this paper, we incorporate nonlocal delay into a threespecies food chain model with Michaelis-Menten functional response to represent a delay due to the gestation of the predator. The conditions, under which the spatial homogeneous equilibria are asymptotically stable, are given by using the Lyapunov functional.

We now summarize the ecological meanings of our theoretical results. Firstly, the positive equilibrium $E^{*}$ of system (1)-(3) exists under the high birth rate of the prey $\left(a_{1}\right)$ and low death rates $\left(a_{2}\right.$ and $\left.a_{3}\right)$ of predator and top predator. $E^{*}$ is globally stable if the intraspecific competition $a_{11}$ is neither too big nor too small and the maximum harvest rates $a_{12}, a_{23}$ are small enough. Secondly, the semi-trivial equilibrium $E_{2}$ of system (1)-(3) exists if the birth rate of the prey $\left(a_{1}\right)$ is high, death rate of predator $a_{2}$ is low, and the death rate $\left(a_{3}\right)$ exceeds the conversion rate from predator to top predator $\left(a_{32}\right) . E_{2}$ is globally stable if the maximum harvest rates $a_{12}, a_{23}$ are small and the intra-specific competition $a_{11}$ is neither too big nor too small. Thirdly, system (1)-(3) has only one semi-trivial equilibrium $E_{1}$ when the death rate $\left(a_{2}\right)$ exceeds the conversion rate from prey to predator $\left(a_{21}\right) . E_{1}$ is globally stable if intra-specific competitions $\left(a_{11}, a_{22}\right.$, and $\left.a_{33}\right)$ are strong. Finally, $E_{0}$ is unstable and the non-stability of trivial equilibrium tells us that not all of the populations go to extinction. Furthermore, our main results imply that the nonlocal delay is harmless for stabilities of all non-negative steady states of system (1)-(3).

There are still many interesting and challenging problems with respect to system (1)-(3), for example, the permanence and stability of periodic solution or almost periodic solution. These problems are clearly worthy for further investigations.

\section{Acknowledgments}

This work is partially supported by PRC Grants NSFC 11102076 and 11071209 and NSF of the Higher Education Institutions of Jiangsu Province (12KJD110002 and 12KJD110008).

\section{References}

[1] R. Xu and M. A. J. Chaplain, "Persistence and global stability in a delayed predator-prey system with Michaelis-Menten type functional response," Applied Mathematics and Computation, vol. 130, no. 2-3, pp. 441-455, 2002.

[2] H. F. Huo and W. T. Li, "Periodic solution of a delayed predatorprey system with Michaelis-Menten type functional response," Journal of Computational and Applied Mathematics, vol. 166, no. 2, pp. 453-463, 2004.

[3] Z. Lin and M. Pedersen, "Stability in a diffusive food-chain model with Michaelis-Menten functional response," Nonlinear Analysis: Theory, Methods \& Applications, vol. 57, no. 3, pp. 421433, 2004.

[4] B. X. Dai, N. Zhang, and J. Z. Zou, "Permanence for the Michaelis-Menten type discrete three-species ratio-dependent food chain model with delay," Journal of Mathematical Analysis and Applications, vol. 324, no. 1, pp. 728-738, 2006.

[5] N. F. Britton, "Spatial structures and periodic travelling waves in an integro-differential reaction-diffusion population model," SIAM Journal on Applied Mathematics, vol. 50, no. 6, pp. 1663$1688,1990$.

[6] S. A. Gourley and N. F. Britton, "Instability of travelling wave solutions of a population model with nonlocal effects," IMA Journal of Applied Mathematics, vol. 51, no. 3, pp. 299-310, 1993.

[7] X. Zhang and R. Xu, "Traveling waves of a diffusive predatorprey model with nonlocal delay and stage structure," Journal of Mathematical Analysis and Applications, vol. 373, no. 2, pp. 475484, 2011.

[8] Z. C. Wang, W. T. Li, and S. G. Ruan, "Existence and stability of traveling wave fronts in reaction advection diffusion equations with nonlocal delay," Journal of Differential Equations, vol. 238, no. 1, pp. 153-200, 2007.

[9] S. A. Gourley and S. G. Ruan, "Convergence and travelling fronts in functional differential equations with nonlocal terms: a competition model," SIAM Journal on Mathematical Analysis, vol. 35, no. 3, pp. 806-822, 2003.

[10] X. H. Lai and T. X. Yao, "Exponential stability of impulsive delayed reaction-diffusion cellular neural networks via Poincaré integral inequality," Abstract and Applied Analysis, vol. 2013, Article ID 131836, 10 pages, 2013.

[11] R. Xu and Z. E. Ma, "Global stability of a reaction-diffusion predator-prey model with a nonlocal delay," Mathematical and Computer Modelling, vol. 50, no. 1-2, pp. 194-206, 2009.

[12] Z. Li, F. D. Chen, and M. X. He, "Asymptotic behavior of the reaction-diffusion model of plankton allelopathy with nonlocal delays," Nonlinear Analysis: Real World Applications, vol. 12, no. 3, pp. 1748-1758, 2011.

[13] Y. Kuang and H. L. Smith, "Global stability in diffusive delay Lotka-Volterra systems," Differential and Integral Equations, vol. 4, no. 1, pp. 117-128, 1991. 
[14] Z. G. Lin, M. Pedersen, and L. Zhang, "A predator-prey system with stage-structure for predator and nonlocal delay," Nonlinear Analysis: Theory, Methods \& Applications, vol. 72, no. 3-4, pp. 2019-2030, 2010.

[15] R. Xu, "A reaction-diffusion predator-prey model with stage structure and nonlocal delay," Applied Mathematics and Computation, vol. 175, no. 2, pp. 984-1006, 2006.

[16] S. S. Chen and J. P. Shi, "Stability and Hopf bifurcation in a diffusive logistic population model with nonlocal delay effect," Journal of Differential Equations, vol. 253, no. 12, pp. 3440-3470, 2012.

[17] H. I. Freedman, Deterministic Mathematical Models in Population Ecology, vol. 57 of Monographs and Textbooks in Pure and Applied Mathematics, Marcel Dekker, New York, NY, USA, 1980.

[18] P. A. Abrams and L. R. Ginzburg, "The nature of predation: prey depedent, ratio dependent or neither," Trends in Ecology and Evolution, vol. 15, no. 8, pp. 337-341, 2000.

[19] D. L. DeAngelis, R. A. Goldstein, and R. V. O’Neill, "A model for trophic iteration," Ecology, vol. 56, no. 4, pp. 881-892, 1975.

[20] C. V. Pao, "Dynamics of nonlinear parabolic systems with time delays," Journal of Mathematical Analysis and Applications, vol. 198, no. 3, pp. 751-779, 1996.

[21] F. Xu, Y. Hou, and Z. G. Lin, "Time delay parabolic system in a predator-prey model with stage structure," Acta Mathematica Sinica, vol. 48, no. 6, pp. 1121-1130, 2005 (Chinese).

[22] P. Y. H. Pang and M. X. Wang, "Strategy and stationary pattern in a three-species predator-prey model," Journal of Differential Equations, vol. 200, no. 2, pp. 245-273, 2004.

[23] D. Henry, Geometric Theory of Semilinear Parabolic Equations, vol. 840 of Lecture Notes in Mathematics, Springer, New York, NY, USA, 1981.

[24] K. J. Brown, P. C. Dunne, and R. A. Gardner, "A semilinear parabolic system arising in the theory of superconductivity," Journal of Differential Equations, vol. 40, no. 2, pp. 232-252, 1981.

[25] S. A. Gourley and J. W. H. So, "Dynamics of a food-limited population model incorporating nonlocal delays on a finite domain," Journal of Mathematical Biology, vol. 44, no. 1, pp. 4978, 2002 . 


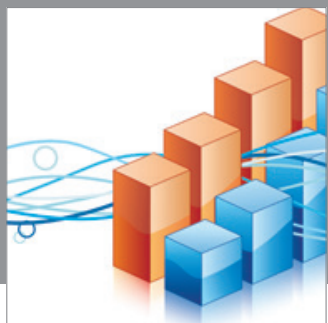

Advances in

Operations Research

mansans

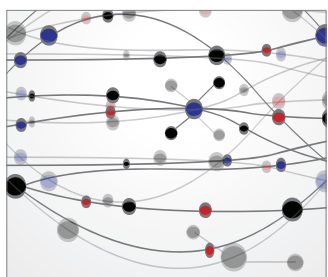

The Scientific World Journal
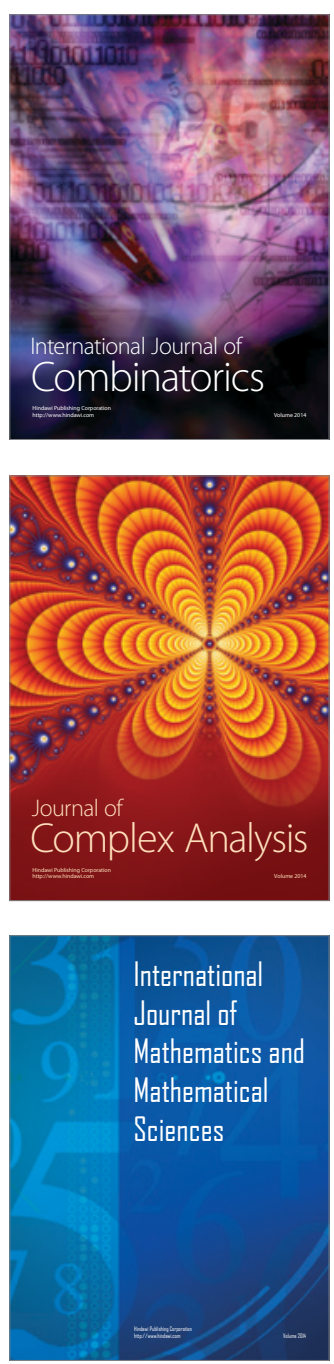
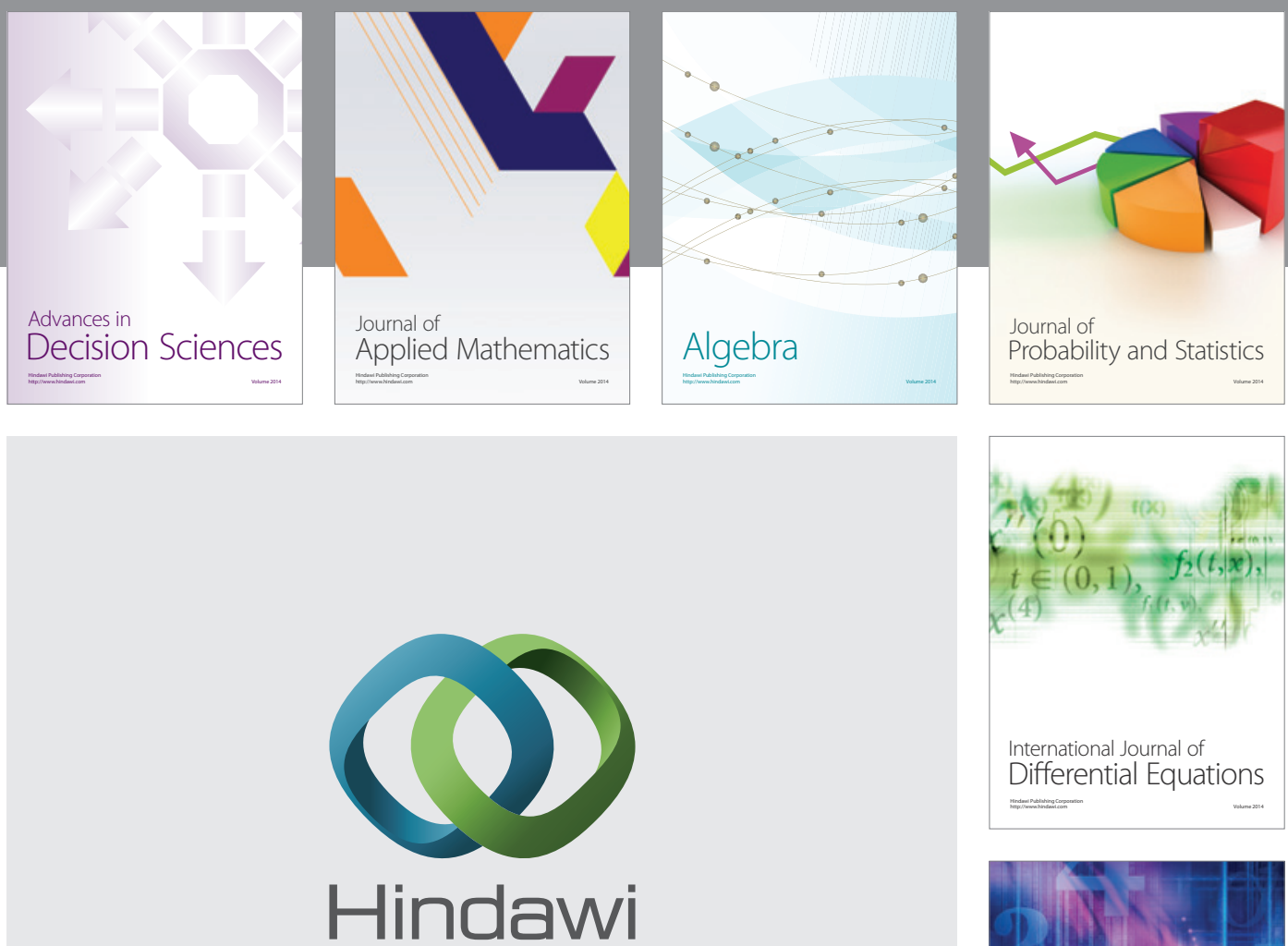

Submit your manuscripts at http://www.hindawi.com
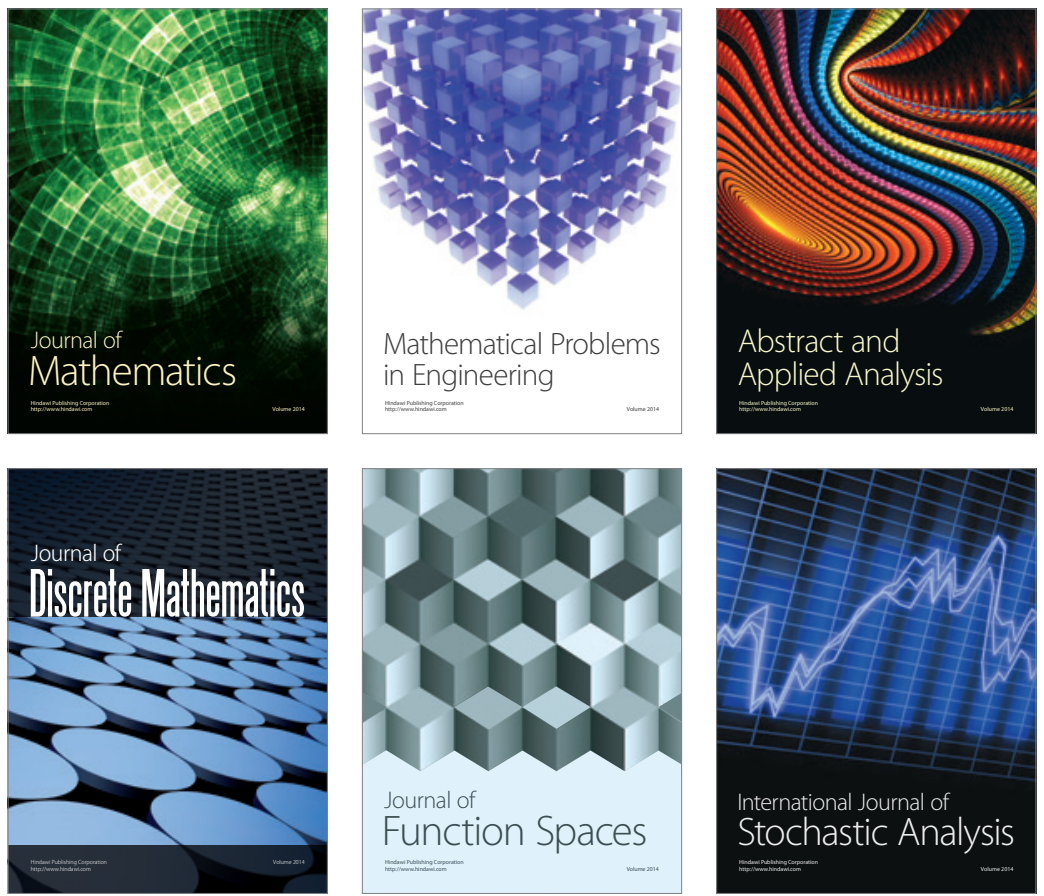

Journal of

Function Spaces

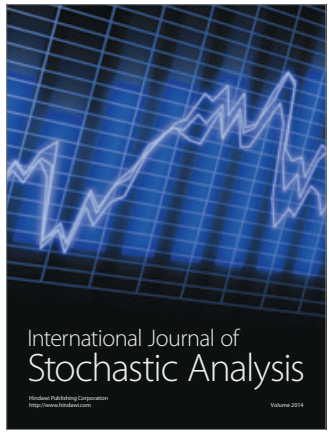

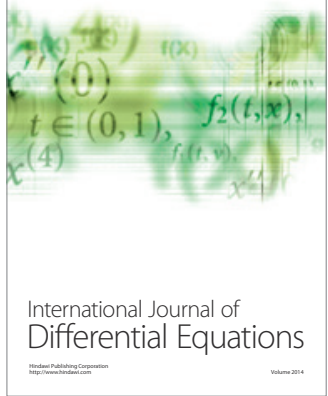
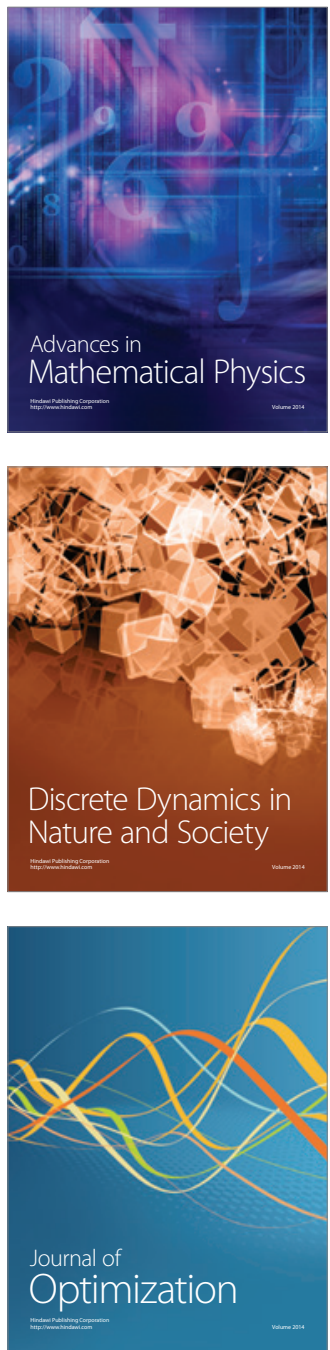